\title{
The clinical effectiveness of intra-articular corticosteroids for arthritis of the lower limb in juvenile idiopathic arthritis: a systematic review
}

Heidi Jennings ${ }^{1}$, Kym Hennessy ${ }^{1}$ and Gordon J Hendry ${ }^{1,2^{*}}$

\begin{abstract}
Background: Juvenile Idiopathic Arthritis (JIA) commonly affects joints of the lower limb including the knee, ankle, subtalar and other foot joints. Intra-articular corticosteroid injections (IACls) are considered to be effective for short-term relief of synovitis, however, there appears to be a significant lack of published evidence from comparative effectiveness studies. The aim of this study was to identify and critically appraise the evidence for the efficacy of lower limb IACls in children/adolescents with JIA.
\end{abstract}

Methods: Studies were identified in databases Medline, Embase, CINAHL, AMED, PEDro, the Cochrane Library and TRIP, with no date restrictions. The primary search terms 'juvenile idiopathic arthritis', 'lower limb', 'knee'; 'ankle', 'foot' and 'intra-articular steroid injections' and related synonyms were used to develop a comprehensive pragmatic literature search strategy. Included studies were quantitative longitudinal design such as randomised controlled trials, pseudo-randomised and non-randomised experimental studies, cohort studies, and case-control studies. All outcomes measures were subject to analysis. Quality assessment was conducted using the Cochrane Collaboration criteria with additional criteria for sample population representativeness, quality of statistical analysis and compliant intervention use and presence of co-interventions. Qualitative data synthesis was conducted for the outcome domains. Meta-analyses were not possible as multiple randomised controlled trials for outcome measures were not available. Levels of evidence were assigned to each outcome measure.

Results: The inclusion criteria were met by twenty-one studies. One study had high quality for internal validity and nine studies had high quality for external validity. No studies had high quality for both internal and external validity. Four outcome domains were identified. There was weak evidence for IACls decreasing clinical signs and symptoms in the lower leg, improving joint range of motion, decreasing leg length discrepancy, and for imaging techniques detecting the effects of IACIs.

Conclusions: There is some weak evidence for the efficacy of IACls improving certain outcome measures. However, there is also some inconclusive evidence due to a lack of quality studies. More high quality evidence is necessary to definitely determine the efficacy of IACIs for JIA in the lower leg.

Keywords: Juvenile idiopathic arthritis, Intra-articular injection, Steroid injection, Systematic review, Lower limb, Knee, Ankle, Foot

\footnotetext{
* Correspondence: gordon.hendry@gcu.ac.uk

'School of Science \& Health, University of Western Sydney, Penrith, NSW, Australia

${ }^{2}$ Institute for Applied Health Research/School of Health and Life Sciences, Glasgow Caledonian University, Glasgow G4 OBA, UK
}

\section{Biomed Central}

(c) 2014 Jennings et al.; licensee BioMed Central Ltd. This is an Open Access article distributed under the terms of the Creative Commons Attribution License (http://creativecommons.org/licenses/by/2.0), which permits unrestricted use, distribution, and reproduction in any medium, provided the original work is properly credited. The Creative Commons Public Domain Dedication waiver (http://creativecommons.org/publicdomain/zero/1.0/) applies to the data made available in this article, unless otherwise stated. 


\section{Background}

Juvenile Idiopathic Arthritis (JIA) is the most common rheumatic disorder in children, affecting approximately 1 in 1000 Australian children [1]. Lower limb arthritis is common in JIA regardless of disease classification, with the knee joint affected most frequently at disease onset (40-60\% of cases) followed by the ankle (20-30\% of cases) $[2,3]$. Persistent synovitis in these joints is associated with pain, reduced joint ranges of motion, deformities, gait disruption, and poor functional status [4-7]. Moreover, radiographic progression of the disease occurs early, and if not addressed may result in permanent joint destruction and poor functional outcomes $[8,9]$.

The primary aim of medical management in JIA is to suppress inflammation prior to the development of irreparable joint damage and functional limitation [10]. Recent improvements in the medical management of JIA have led to a greater emphasis on early diagnosis of JIA, early detection of synovitis, and subsequent prompt and aggressive systemic medical intervention via disease-modifying and/or biologic regimens to control the inflammatory disease process [11]. The introduction of such regimens have led to a relative increase in the number of patients achieving disease improvements characterised by low disease activity or remission [12]. However, in spite of promising signs of improvement, children may experience refractory disease, characterised by continued persistent synovitis, and/or serious adverse effects including hepato-toxicity and immuno-deficiency following administration of methotrexate and etanercept [13].

Intra-articular corticosteroid injections (IACIs) are often used as the main form of management in milder oligoarticular JIA, and also as an adjunct to systemic therapy in other JIA subtypes where localised synovitis is restricted to a single or a small number of joints $[13,14]$. IACIs are administered to induce rapid relief of symptoms through resolution of localised synovitis, and in some cases may prevent the need for escalation of systemic therapy and associated increased risk of serious side-effects [14]. It is acknowledged that IACIs are also associated with adverse events, however, these may be relatively mild compared to those associated with systemic disease-modifying antirheumatic drugs (DMARDs) or biologics. These may include subcutaneous atrophy, hypopigmentation and/or post-injection pain $[14,15]$. Several inconsistencies have been highlighted in studies of IACIs such as variable injection techniques, inclusion criteria, post-injection procedures, outcome measures, and follow-up period $[14,16,17]$.

Previous systematic reviews have been conducted but they are now outdated and new studies of IACIs have been published since they were completed. The Cochrane Collaboration recommends that systematic reviews are updated biannually, and the most relevant and recent review was published seven years ago [18]. Two systematic reviews have been conducted to evaluate the evidence for IACIs in JIA; one which focused on IACIs for temporomandibular joint (TMJ) arthritis only [16]; and another which reviewed studies on lower limb IACIs for children with JIA and/or adults with rheumatoid arthritis (RA). However, ultimately they did not include any studies of JIA [17]. The recent systematic review of IACIs for TMJ arthritis [16] may not be directly relevant to this work as IACIs administered in the joints of the lower limb appear to be less efficacious than those administered in upper limb and non-weight-bearing joints [19]. Moreover IACIs administered to the knee joints appear to be more efficacious than those administered to the small joints in the foot, where the response is less predictable [19]. Two other reviews of the overall medical management of JIA adopted systematic approaches to conduct their literature search, but their restrictive inclusion criteria retrieved only two small randomised studies concerning IACIs and only limited conclusions were drawn $[10,20]$. One other systematic review attempted to appraise the evidence for knee joint IACIs for a range of arthritis conditions including JIA, osteoarthritis (OA) and RA [21]. However, methodological problems were identified with this review including a single joint focus (knee), adult arthritis predominance for studies included, and violations of the detailed inclusion criteria. Narrative reviews have also been conducted and have provided useful summaries of the literature concerning IACIs in JIA [14,22]. However, such reviews are often reinforced with the authors' expert opinions and clinical experience and as a result may be vulnerable to bias [23].

Most research concerning IACIs efficacy to date appears to focus predominantly on the knee joint only, or a combination of several joints which differ in terms of anatomical complexity, and may include weight-bearing/ non-weight-bearing joints which could impact on IACI efficacy. The reviews which have been published to date fail to account for the efficacy of IACIs for synovitis relief in other important lower limb joints, and thus the findings and conclusions drawn from these reviews are not necessarily generalizable. Lastly, it should be noted that standard clinical practice has changed in recent years with a greater emphasis being placed on image-guidance to aid the administration of intra-articular injection therapies [24-26]. This advancement of clinical practice may have led to improvements in the effectiveness of IACIs on the basis of improved steroid placement accuracy and thus fewer reports of adverse events [24-26]. The emergence of further evidence, introduction of new technologies and changing clinical practices has raised important new questions concerning lower limb IACI efficacy in JIA. Accordingly, the aim of this review is to systematically identify and critically evaluate the clinical effectiveness of IACIs administered to joints of the lower limb in people with JIA. 


\section{Methods}

\section{Search strategy}

A detailed electronic database search of the literature was performed using the following databases: Medline, Embase, CINAHL, AMED, PEDro, the Cochrane Library, and TRIP. Reference lists of eligible studies identified by the electronic database search were searched by hand to identify studies not initially found in the electronic search. Personal reference lists of the reviewers were also hand searched to identify any studies known to the reviewers that may not have been identified by the initial search strategy. The following search terms 'juvenile idiopathic arthritis', 'lower limb', 'knee', 'ankle', 'foot', and 'intra-articular steroid injections' and related synonyms were used to develop a comprehensive pragmatic literature search strategy. Standard MeSH terms were utilised where possible or an appropriate text word was adopted. Boolean operators (such as 'explode', 'OR', '*', '\$' and 'AND') were used. The full search strategy is outlined in Additional file 1.

\section{Study inclusion criteria}

Studies included were of a quantitative longitudinal design, such as randomised controlled trials (RCTs), pseudorandomised and non-randomised experimental studies, cohort studies, and case-control studies. Studies of single cases were excluded. Studies reporting children, adolescents and adults who have JIA were included. Studies of IACIs as the single primary intervention, in combination with systemic medications, and/or in combination with nonpharmacological interventions were included. All outcome measures/variables were subject to analysis. Literature was limited to full text published articles available in English due to language barriers of both independent reviewers. No restriction was imposed on year of publication.

Study abstracts found from the electronic database search and by hand searching were reviewed for eligibility based upon the above inclusion criteria. Two independent reviewers $(\mathrm{HJ}$ and $\mathrm{KH})$ selected abstracts which appeared to meet the inclusion criteria. Full text articles of the selected abstracts were obtained and compared against the inclusion criteria. The full text, original research articles meeting the inclusion criteria were assessed for quality.

\section{Quality assessment}

Quality assessment was conducted by two independent reviewers $(\mathrm{HJ}$ and $\mathrm{KH})$ using an adaption of The Cochrane Collaboration tool for assessing risk of bias as previously described [18,27]. For randomised studies the internal validity criteria included sequence generation, allocation concealment, blinding of participants and personnel, blinding of outcome assessment, incomplete outcome data, selective outcome reporting, and selective outcome reporting. Whereas for non-randomised studies, adapted criteria were adopted that did not include criteria that was relevant to randomised studies only. Further internal validity criteria were also included to determine presence of cointerventions, intervention compliance, and if proper statistical analysis was conducted. External validity criteria were also included to determine restrictiveness of the inclusion and exclusion criteria, and to determine if the sample population was representative of the general population with JIA. For a study to achieve a high quality score overall, all included domains had to be scored independently as high quality by both reviewers. A study that was independently rated with $\geq 1$ domain scored as low quality was rated as low quality overall. Any disagreement was resolved by a third reviewer (GJH).

\section{Data extraction/evidence grading}

The data extraction/evidence grading system was agreed upon by the co-authors a priori and is outlined as follows. Due to a lack of multiple RCTs for specific outcomes measures, meta-analysis was not possible and as such qualitative data synthesis was conducted. Once extracted data was analysed and synthesised, an evidence rating was assigned according to previously published criteria [28] (Table 1). Once studies were rated for quality, they were grouped according to the outcomes measured and then the evidence grading system was adopted to assign levels of evidence for each outcome. This evidence grading criteria considers both quality and quantity of studies as well as the consistency of the findings for each outcome [28].

\section{Results}

Using the detailed search strategy, a total of 568 articles were retrieved. The inclusion criteria were met by twenty-one studies (see Figure 1), and a description of each study is presented in Table 2. The majority of the studies were observational in nature, with only one RCT identified for inclusion. Therefore, meta-analyses were not possible as were originally intended. One study had high quality internal validity and twenty had low quality internal validity. Nine studies had high quality external validity and twelve had low quality external validity. There were no studies with both high quality internal and external validity (Table 3 ). From the assessed studies, nine outcome domains were identified which included tenderness/pain, swelling, synovitis, effusion, hyperthermia, sustained 'clinical response', joint range of motion (ROM), length discrepancy (leg length discrepancy or joint circumference), and imaging detectable outcomes. Leg length discrepancy (LLD) was defined as unequal length of the lower limb from the knee distally, as a result of knee synovitis and/or tibial growth disturbances. Qualitative syntheses of results are outlined below and presented in Table 4 . 
Table 1 Evidence rating criteria (adapted from Ariens et al, 2000 [28])

\begin{tabular}{ll}
\hline Strong & At least $\mathbf{2}$ studies of high quality with consistent findings (agreement of $>\mathbf{7 5 \%}$ of studies) \\
\hline Moderate & 1 high quality study and at least 2 low-quality studies with consistent findings (agreement of $>75 \%$ of studies) \\
Weak & At least 2 low-quality studies with consistent findings (agreement of $>75 \%$ of studies) \\
Inconclusive & Insufficient and/or conflicting studies \\
\hline
\end{tabular}

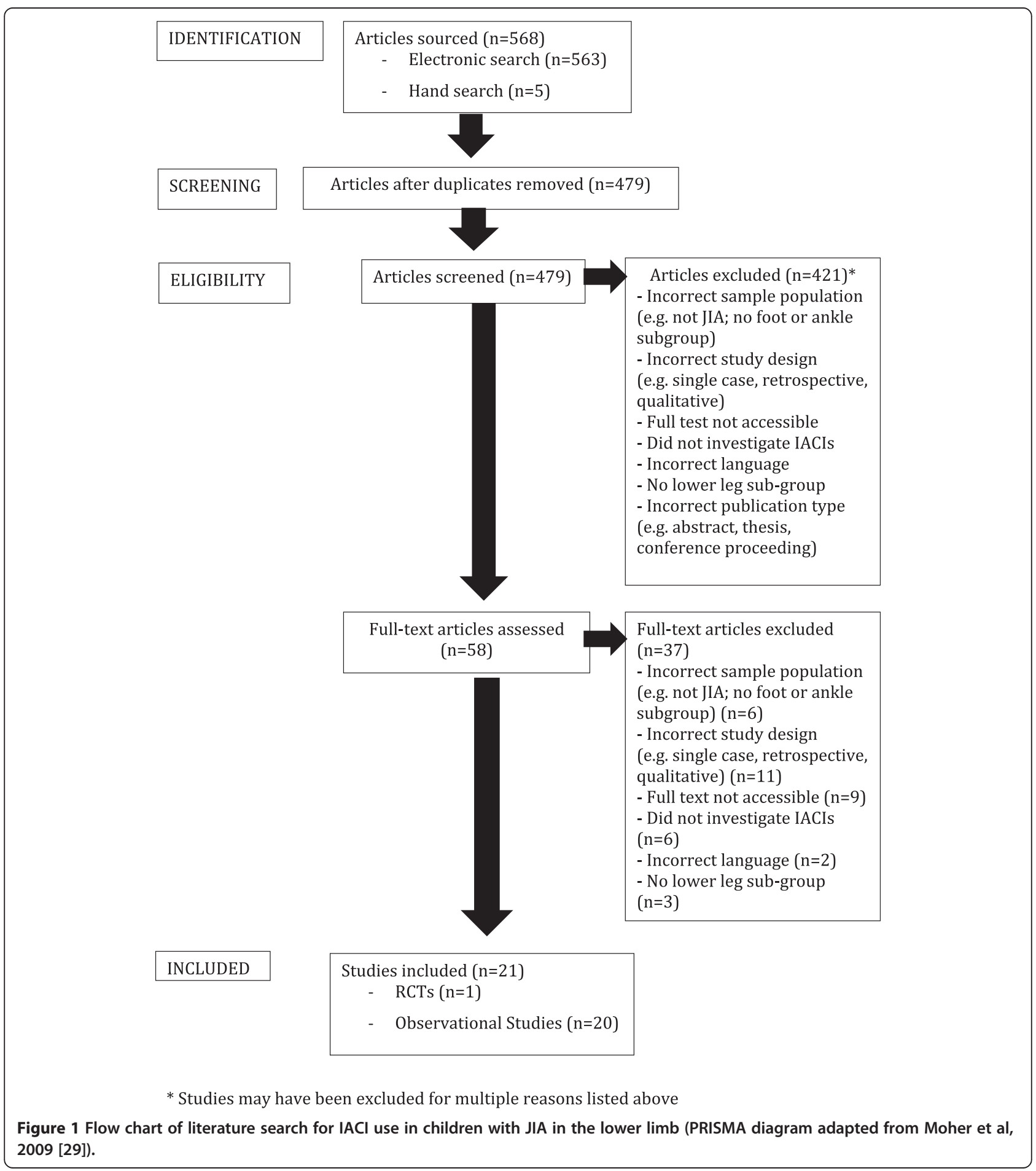


Table 2 Description of included studies

\begin{tabular}{|c|c|c|c|c|c|c|}
\hline Author, year & $\begin{array}{l}\text { Study } \\
\text { type }\end{array}$ & Participant description & $\begin{array}{l}\text { No. entered/ } \\
\text { completed study }\end{array}$ & Follow-up period & $\begin{array}{l}\text { Intervention (including } \\
\text { post-injection procedure) }\end{array}$ & Outcome measures \\
\hline \multirow{5}{*}{$\begin{array}{l}\text { Balogh } \\
\text { et al, [30] }\end{array}$} & \multirow[t]{5}{*}{$\mathrm{RCT}$} & \multirow{3}{*}{$\begin{array}{l}\text { Fulfilment of EULAR/WHO } \\
\text { Oslo Criteria for JCA. }\end{array}$} & \multirow[t]{5}{*}{ 23/23 (23 knees) } & \multirow[t]{5}{*}{$1,3,7$ and 42 days } & $\mathrm{TH}$ in $11 / 23$ & \multirow{5}{*}{$\begin{array}{l}\text { Knee Joint Circumference (cm); } \\
\text { Knee Joint Flexion (degrees). }\end{array}$} \\
\hline & & & & & BM in $12 / 23$ & \\
\hline & & & & & (Dose not recorded) & \\
\hline & & Pauciarticular form of disease. & & & \multirow[t]{2}{*}{ Post-injection: Not specified. } & \\
\hline & & Co-interventions: Not specified & & & & \\
\hline \multirow[t]{2}{*}{$\begin{array}{l}\text { Al-wahadneh, } \\
\text { [31] }\end{array}$} & \multirow[t]{2}{*}{ OBS } & $\begin{array}{l}\text { Failure to respond to NSAIDs } \\
\text { with/without slow acting } \\
\text { anti-inflammatories. }\end{array}$ & \multirow[t]{2}{*}{ 24/24 (30 knee joints) } & \multirow[t]{2}{*}{$3,6,9,12$ and 24 months. } & $\begin{array}{l}\text { MA } 1 \mathrm{mg} / \mathrm{kg} / \mathrm{joint} \text { mixed with } 1 \mathrm{cc} \\
1 \% \text { lidocaine without adrenaline. }\end{array}$ & \multirow{2}{*}{$\begin{array}{l}\text { Sustained Clinical Response: } \\
\text { 'Active Inflammation' (joint } \\
\text { effusion and heat and } \\
\text { tenderness/pain with/without } \\
\text { correction of deformity) }\end{array}$} \\
\hline & & $\begin{array}{l}\text { Co-interventions: 10/24: MTX } \\
\text { (10 mg/kg/wk), 24/24: Naproxen } \\
\text { (10-20 mg/kg/day), 24/24: NSAIDs, } \\
\text { 11/24: Prednisolone (0.25 mg/kg/day) }\end{array}$ & & & $\begin{array}{l}\text { Post-injection: Immobilisation } 24 \\
\text { hrs post injection before } \\
\text { commencing physiotherapy. }\end{array}$ & \\
\hline \multirow[t]{4}{*}{$\begin{array}{l}\text { Allen et al., } \\
\text { [32] }\end{array}$} & \multirow[t]{4}{*}{ OBS } & $\begin{array}{l}<16 \text { yrs of age at onset of } \\
\text { chronic arthritis } 29 / 40 \\
\text { pauciarticular onset JRA }\end{array}$ & \multirow[t]{4}{*}{$\begin{array}{l}\text { 40/40 (53 knees) } 4 \\
\text { patients lost to follow-up. }\end{array}$} & \multirow[t]{4}{*}{$3,6,12$ and 24 months. } & $\begin{array}{l}20-40 \mathrm{mg} \text { TH with } 1 \% \text { xylocaine } \\
\text { without epinephrine infiltration. }\end{array}$ & $\begin{array}{l}\text { Sustained Clinical Response: } \\
\text { 'Active }\end{array}$ \\
\hline & & $\begin{array}{l}\text { 4/40 seronegative enthesopathy- } \\
\text { arthropathy syndrome }\end{array}$ & & & Post-injection: Not specified & \multirow{3}{*}{$\begin{array}{l}\text { Inflammation'(Joint effusion } \\
\text { and heat and tenderness, } \\
\text { with or without complete } \\
\text { correction of deformity) }\end{array}$} \\
\hline & & $6 / 40$ psoriatic arthritis & & & & \\
\hline & & $\begin{array}{l}\text { Co-interventions: } 100 \% \text { - acetylsalicylic acid } \\
\text { for at least } 3 \text { months; } 30 \%-1 \text { NSAID; } \\
22.5 \%-2 \text { NSAIDs; } 20 \%-\geq 3 \text { NSAIDs; } \\
3 \text { patients previously on oral prednisone; } \\
1 \text { patient on hydroxychloroquine; } 4 \text { patients } \\
\text { prior corticosteroid injection (other than TH); } \\
\text { all patients maintained on the same } \\
\text { NSAIDs post-injection. }\end{array}$ & & & & \\
\hline \multirow{4}{*}{$\begin{array}{l}\text { Beukelman } \\
\text { et al., [26] }\end{array}$} & \multirow[t]{4}{*}{ OBS } & Definitive JIA diagnosis based on criteria. & \multirow[t]{4}{*}{ 38/38 (55 STJ injections) } & \multirow{4}{*}{$\begin{array}{l}1-30 \text { weeks } \\
\text { (median }=6 \text { weeks) }\end{array}$} & TH in 13/38TA in 24/38 & \multirow{4}{*}{$\begin{array}{l}\text { Sustained Clinical Response: } \\
\text { STJ Eversion/Inversion and } \\
\text { pain and gait abnormalities }\end{array}$} \\
\hline & & $\begin{array}{l}\text { Decreased foot inversion or } \\
\text { eversion on physical examination }\end{array}$ & & & $\begin{array}{l}\text { (Medication and dose not } \\
\text { recorded in 1/39 injection) }\end{array}$ & \\
\hline & & \multirow{2}{*}{$\begin{array}{l}\text { Co-interventions: 14/38: MTX, 3/38: } \\
\text { TNF-alpha inhibitor, 3/38: TNF-alpha } \\
\text { inhibitor and MTX. }\end{array}$} & & & $\begin{array}{l}\text { TA used when TH was } \\
\text { commercially unavailable. }\end{array}$ & \\
\hline & & & & & $\begin{array}{l}\text { Post-injection: Non-weight bearing } \\
24 \text { hrs post-injection. Normal } \\
\text { activity resumed afterwards. }\end{array}$ & \\
\hline \multirow{4}{*}{$\begin{array}{l}\text { Cahill } \\
\text { et al., [25] }\end{array}$} & \multirow[t]{3}{*}{ OBS } & Clinical signs of STJ inflammation & $38 / 38 ; 24 / 38$ & $2-3$ months & $0.5-1 \mathrm{ml}, 20 \mathrm{mg} / \mathrm{ml} \mathrm{TH}$ & Increased STJ \\
\hline & & Referral for image-guided IACI. & \multirow{2}{*}{$\begin{array}{l}\text { single STJ; } 4 / 38 \text { bilateral } \\
\text { STJ; } 3 / 38 \text { subsequent } \\
\text { contra-lateral STJ; } 7 / 38 \text { at } \\
\text { least one repeated STJ. }\end{array}$} & \multirow{2}{*}{$\begin{array}{l}\text { (active disease) } 6 \text { months } \\
\text { (without active disease) }\end{array}$} & Post-injection: Not specified. & \multirow{3}{*}{$\begin{array}{l}\text { inversion and eversion: Normal } \\
\text { ROM, without pain and limping }\end{array}$} \\
\hline & & Co-interventions: Not specified. & & & & \\
\hline & OBS & Pauci-articular onset JCA. & 23/23 (86 knees) & 3,6 and 12 months & & \\
\hline
\end{tabular}


Table 2 Description of included studies (Continued)

\begin{tabular}{|c|c|c|c|c|c|c|}
\hline \multirow{3}{*}{\multicolumn{2}{|c|}{$\begin{array}{l}\text { Earley } \\
\text { et al., [33] }\end{array}$}} & \multicolumn{3}{|l|}{ Painful swollen knee with poor function. } & \multirow{3}{*}{$\begin{array}{l}\text { TH } 20 \text { mg for children weighing } \\
<20 \mathrm{~kg}(63 \mathrm{knees} \text { ) OR for children } \\
>20 \mathrm{mg} 40 \mathrm{mg} \text { (20 knees) } \\
\text { Post-injection: Not specified. }\end{array}$} & \multirow{3}{*}{$\begin{array}{l}\text { Sustained Clinical Response: } \\
\text { Soft tissue swelling and joint } \\
\text { effusion and degree of flexion } \\
\text { contracture and degree of } \\
\text { valgus deformity. }\end{array}$} \\
\hline & & \multicolumn{3}{|l|}{$\begin{array}{l}\text { Not respondent to at least } 3 \\
\text { months of conventional treatment. }\end{array}$} & & \\
\hline & & \multicolumn{3}{|l|}{$\begin{array}{l}\text { Co-interventions: All patients were on } \\
\text { at least one NSAID and physiotherapy } \\
\text { and splinting, } 3 / 23 \text { on gold, } 1 / 23 \\
\text { on prednisolone }\end{array}$} & & \\
\hline \multirow{4}{*}{$\begin{array}{l}\text { Eberhard } \\
\text { et al., [34] }\end{array}$} & \multirow[t]{4}{*}{ OBS } & JRA diagnosis based on ACR criteria. & \multirow{4}{*}{$\begin{array}{l}\text { 85/85 (51/99 received TH } \\
\text { and 48/99 received TA; } 14 \\
\text { patients received both) }\end{array}$} & \multirow{4}{*}{$\begin{array}{l}2 \text { weeks then every } 3 \\
\text { months for a minimum } \\
\text { for } 15 \text { months. }\end{array}$} & \multirow{4}{*}{$\begin{array}{l}\text { TH: } 40 \text { mg (knee), } 30 \text { mg (ankle). } \\
\text { TA: } 80 \text { mg (knee), } 60 \text { mg (ankles) } \\
\text { Post injection: Minimal activity } \\
\text { for } 24 \text { hrs post-injection. }\end{array}$} & \multirow{4}{*}{$\begin{array}{l}\text { Sustained Clinical Response: } \\
\text { Non-bony swelling and } \\
\text { (if no swelling) limitation } \\
\text { ROM pain on motion or } \\
\text { joint tenderness. }\end{array}$} \\
\hline & & \multirow{2}{*}{$\begin{array}{l}\text { Co-interventions: TH group: } 33 / 51 \\
\text { NSAIDs; } 16 / 51 \text { MTX; } 9 / 51 \text { sulfasalzine; } \\
\text { 3/51 prednisone + etanercept; } \\
\text { 14/51 no medication. }\end{array}$} & & & & \\
\hline & & & & & & \\
\hline & & $\begin{array}{l}\text { TA group: } 33 / 51 \text { NSAIDs; } 12 / 51 \text { MTX; } \\
\text { 2/51 sulfasalazine; } 4 / 51 \text { etanercept; } \\
\text { 2/51 prednisone; } 2 / 51 \text { leflunomide; } \\
15 / 51 \text { no medication. }\end{array}$ & & & & \\
\hline \multirow[t]{14}{*}{ Eich et al., [35] } & \multirow[t]{14}{*}{ OBS } & JCA diagnosis based on & \multirow[t]{14}{*}{ 15/15 (11 knees) } & \multirow{14}{*}{$\begin{array}{l}\text { Clinical and US assessment: } \\
1 \text { week and } 1 \text { month } \\
\text { MRI: } 1 \text { month }\end{array}$} & \multirow{14}{*}{$\begin{array}{l}\text { TH: } 40 \text { mg (knee) } \\
\text { Post-injection: Not specified. }\end{array}$} & Pain \\
\hline & & EULAR criteria. & & & & \\
\hline & & \multirow{2}{*}{$\begin{array}{l}\text { Failure of systemic therapy } \\
\text { and physiotherapy. }\end{array}$} & & & & \multirow{5}{*}{$\begin{array}{l}\text { Swelling } \\
\text { Hyperthermia } \\
\text { Limited ROM } \\
\text { Leg-length discrepancy } \\
\text { MRI: Joint effusion }\end{array}$} \\
\hline & & & & & & \\
\hline & & Local growth disturbances. & & & & \\
\hline & & Popliteal cyst in affected knee. & & & & \\
\hline & & $\begin{array}{l}\text { With/without complete } \\
\text { deformity correction. }\end{array}$ & & & & \\
\hline & & \multirow[t]{7}{*}{ Co-interventions: Not specified. } & & & & Popliteal cyst \\
\hline & & & & & & $\begin{array}{l}\text { Destruction of articular } \\
\text { cartilage and/or bone }\end{array}$ \\
\hline & & & & & & Destruction of menisci \\
\hline & & & & & & Marrow oedema \\
\hline & & & & & & Avascular necrosis \\
\hline & & & & & & $\begin{array}{l}\text { US: Joint effusion } \\
\text { and/or pannus }\end{array}$ \\
\hline & & & & & & Popliteal cyst \\
\hline \multirow{4}{*}{$\begin{array}{l}\text { Hertzberger-ten } \\
\text { Cate et al., [36] }\end{array}$} & \multirow[t]{4}{*}{ OBS } & Type 1 pauciarticular JCA. & \multirow[t]{4}{*}{ 21/21 (27 knees) } & \multirow[t]{4}{*}{6 months } & \multirow{4}{*}{$\begin{array}{l}\text { TA: } 20 \mathrm{mg} \text { in children weighing } \\
>20 \mathrm{~kg} \text { with } 1 \mathrm{ml} \text { lignocaine. }\end{array}$} & \multirow{4}{*}{$\begin{array}{l}\text { Sustained Clinical Response: } \\
\text { swelling and synovial fluid } \\
\text { and no increased temperature. }\end{array}$} \\
\hline & & Chronic arthritis in $\geq 1$ knee. & & & & \\
\hline & & $\begin{array}{l}\text { No response } \geq 6 \text { months of } \\
\text { conventional treatment. }\end{array}$ & & & & \\
\hline & & $\begin{array}{l}\text { Flexion contracture, } \\
\text { muscle wasting and/or } \\
\text { growth disturbances. }\end{array}$ & & & & \\
\hline
\end{tabular}


Table 2 Description of included studies (Continued)

\begin{tabular}{|c|c|c|c|c|c|c|}
\hline & & $\begin{array}{l}\text { Co-interventions: } 81 \% \text { used splints } \\
\text { at night and } 1 \mathrm{hr} \text { during the day, } \\
50 \% \text { received physiotherapy, } \\
77 \% \text { received NSAIDs. }\end{array}$ & & & $\begin{array}{l}\text { Post-injection: knee passively } \\
\text { flexed and extended several } \\
\text { times to distribute drug. No } \\
\text { advice regarding activity levels. }\end{array}$ & \\
\hline \multirow{4}{*}{$\begin{array}{l}\text { Honkanen } \\
\text { et al., [37] }\end{array}$} & \multirow[t]{4}{*}{ OBS } & JIA diagnosis. & \multirow[t]{4}{*}{ 79/79 (79 knees) } & \multirow[t]{4}{*}{ 6-8 weeks } & MA: in 45/79 (Mean dose & \multirow{4}{*}{$\begin{array}{l}\text { Sustained Clinical } \\
\text { Response: absence/ } \\
\text { presence of 'symptoms' }\end{array}$} \\
\hline & & With/without previous IACI. & & & 1.5 mg/kg) & \\
\hline & & \multirow{2}{*}{$\begin{array}{l}\text { Co-interventions: } 100 \% \text { on NSAID } \\
\text { agents and regular physiotherapy, } \\
17 / 79 \text { on hydroxychloroquine, } \\
\text { sodium aurothiomalate or auranofin } \\
\text { (slow-acting antirheumatics), 5/79 on } \\
\text { alternate day glucocorticoid therapy. }\end{array}$} & & & $\begin{array}{l}\text { TH: in 34/79 (mean } \\
\text { dose } 0.7 \mathrm{mg} / \mathrm{kg})\end{array}$ & \\
\hline & & & & & $\begin{array}{l}\text { Post-injection: Non- weight } \\
\text { bearing for } 24 \text { hrs. }\end{array}$ & \\
\hline \multirow[t]{5}{*}{$\begin{array}{l}\text { Huppertz } \\
\text { et al., [38] }\end{array}$} & \multirow[t]{5}{*}{ OBS } & $\begin{array}{l}\text { Children with chronic arthritis, } \\
\text { not responding to NSAIDs. }\end{array}$ & \multirow[t]{5}{*}{ 21/21(18 knees, 2 ankles) } & \multirow[t]{5}{*}{7,13 weeks } & \multirow{2}{*}{$\begin{array}{l}\text { Knees: } 1 \mathrm{mg} / \mathrm{kg} \text { TH with } \\
20 \mathrm{mg} \text { min dose and } \\
60 \mathrm{mg} \text { maximum dose. }\end{array}$} & Joint Swelling \\
\hline & & \multirow{4}{*}{$\begin{array}{l}\text { Co-interventions: Not pre-specified. } \\
\text { After } 13 \text { weeks, } 10 / 21 \text { had been } \\
\text { treated with concomitant NSAIDs } \\
\text { and } 5 / 21 \text { received chloroquine. }\end{array}$} & & & & \multirow[t]{2}{*}{ Effusion } \\
\hline & & & & & Ankle: a lower dose & \\
\hline & & & & & Post injection: Not specified. & Joint limitation \\
\hline & & & & & & Joint tenderness/pain \\
\hline \multirow{4}{*}{$\begin{array}{l}\text { Laurell } \\
\text { et al., [24] }\end{array}$} & \multirow[t]{4}{*}{ OBS } & JIA diagnosis based on ILR criteria. & \multirow[t]{4}{*}{ 30/30 (40 ankle regions) } & \multirow[t]{4}{*}{4 weeks } & TA $40 \mathrm{mg} / \mathrm{ml}$ & Pain \\
\hline & & Active disease. & & & Post-injection: Not specified. & $\begin{array}{l}\text { US: Synovial hypertrophy } \\
\text { Synovial }\end{array}$ \\
\hline & & $\begin{array}{l}\text { Co-interventions: } 26 \text { patients had } \\
\text { ongoing systemic treatment: } \\
58 \% \text { with MTX }\end{array}$ & & & & hyperaemia \\
\hline & & $\begin{array}{l}\text { 23\% with MTX and biologics } \\
\text { (3 etanercept, } 2 \text { adalimumab, } \\
1 \text { abatacept), } 19 \% \text { with systemic } \\
\text { corticosteroids }\end{array}$ & & & & Joint ROM \\
\hline \multirow{4}{*}{$\begin{array}{l}\text { Lepore } \\
\text { et al., [39] }\end{array}$} & \multirow[t]{4}{*}{ OBS } & JIA patients. & \multirow{4}{*}{$\begin{array}{l}37 / 37 \text { ( } 87 \text { injections } \\
\text { of } 37 \text { knees) }\end{array}$} & \multirow{4}{*}{$\begin{array}{l}7-65 \text { months } \\
\text { (average of } \\
31.3 \text { months) }\end{array}$} & TH: 1 mg/kg (maximum 40 mg) & \multirow{4}{*}{$\begin{array}{l}\text { Sustained Clinical } \\
\text { Response: 'Clinical } \\
\text { signs of inflammation' }\end{array}$} \\
\hline & & $\begin{array}{l}\text { Failure to respond to } 2 \\
\text { months NSAIDs. }\end{array}$ & & & $\begin{array}{l}\text { Post-injection: Advised to } \\
\text { keep child at home for first }\end{array}$ & \\
\hline & & Relapse after full remission period. & & & $\begin{array}{l}24 \text { hrs and to avold physical } \\
\text { exertion and carrying weights. }\end{array}$ & \\
\hline & & $\begin{array}{l}\text { Co-interventions: NSAID use was } \\
\text { discontinued in all patients at } \\
\text { time of local treatment }\end{array}$ & & & & \\
\hline \multirow[t]{3}{*}{$\begin{array}{l}\text { Marti } \\
\text { et al., [19] }\end{array}$} & \multirow[t]{3}{*}{ OBS } & $\begin{array}{l}\text { JIA patients who received } \\
\text { injections and follow } \\
\text { up as in patients. }\end{array}$ & \multirow[t]{3}{*}{$\begin{array}{l}\text { 60/60 (108 knees; } 29 \\
\text { ankles, } 5 \text { STJ and } 3 \\
\text { midfoot) }\end{array}$} & \multirow{3}{*}{$\begin{array}{l}\text { Knee: 1-69 months, } \\
\text { Ankle: } 1-39 \text { months, STJ: } \\
13 \text { months, Midfoot: } \\
\text { 0-3months. Digits: } \\
\text { 18-66 months }\end{array}$} & $\begin{array}{l}\text { TH: } 40 \text { mg (knee, shoulder } \\
\text { and hips); } 20 \text { mg (wrist, } \\
\text { elbow, ankle and STJ); }\end{array}$ & \multirow[t]{3}{*}{$\begin{array}{l}\text { Sustained Clinical Response: } \\
\text { Swelling and effusion } \\
\text { and tenderness/pain }\end{array}$} \\
\hline & & \multirow{2}{*}{$\begin{array}{l}\text { Co-interventions: Non-steroidal } \\
\text { anti-rheumatic drugs, MTX, } \\
\text { Systemic corticosteroids, } \\
\text { Salazopyrine }\end{array}$} & & & $5 \mathrm{~m}$ & \\
\hline & & & & & $\begin{array}{l}\text { TA: } 80 \text { mg (knee, shoulder and } \\
\text { hips); } 40 \text { mg (wrist, elbow, ankle } \\
\text { and STJ); } 10 \text { mg (finger and toes). }\end{array}$ & \\
\hline
\end{tabular}


Papadopoulou OBS Diagnosis JIA based on ILR criteria. et al., [40]

Ravelli
et al.,

et al., [41]

Remedios

et al., [42]

Sherry

et al., [43]

Previous IACls with minimum

follow-up of 6 months.

Co-interventions: $61.8 \%$ of patients received systemic medications including: MTX (56.8\%)

Biologic agents $(9.5 \%)$, Systemic corticosteroids (11.4\%)

\section{OBS JIA diagnosis.}

Initial injection between Feb

1996 and June 1990

Co-interventions: $57 \%$ on NSAIDs,

$24 \%$ on NSAIDs and "2nd line drugs"

OBS Children with JIA presenting

as painful swollen ankles.

Co-interventions: Not Specified

OBS Pauciarticular JCA from ACR criteria. (no specification of second line drugs)

$<7$ years at diagnosis.

Reviewed at rheumatology centres

at University of Washington or

North Carolina (University of

North Carolina/Duke University)

Co-interventions: University of Washington Group: $25 \%$ on DMARD therapy, $44 \%$ with

physical therapy evaluation, $31 \%$ with splints, 0\% with shoe lifts North Carolina Group: 21\% on DMARD therapy, $57 \%$ with physical
220/220 (186 knees, $168 \quad 6$ months ankles, 67 STJ, 14 MTJ and 14 (PJ)

\section{4/94 (66/94- unilateral \\ knee 28/94 bilateral}

knees)

11/11 (13 ankles)

16/16 (15 knees and 6 ankles)

months

$\mathrm{H}: 1 \mathrm{mg} / \mathrm{kg}$ (maximum

$40 \mathrm{mg}$ ) with $0.5 \mathrm{~m}$

lignocaine (2\%).

Post-injection: Rest joints

for 24 hrs

TH: $20 \mathrm{mg}$ with $1 \mathrm{ml} \mathrm{0.5 \%}$

bupivacaine.

Post-injection: Not specified.

Mean follow-up University of University of Washington Washington group: 42 months Group: 20 mg TH within (SD +/- 11).

2 months of diagnosis.

North Carolina group: $\quad$ North Carolina Group: No IACl. 46 months (SD +/- 15)
Sustained Clinical Response: Synovitis

Sustained Clinical Response: Synovitis (clinically assessed) MRI: Pannus

Leg Length Discrepancy (cm)

Thigh circumference discrepancy $(\mathrm{cm})$ 
Table 2 Description of included studies (Continued)

\begin{tabular}{|c|c|c|c|c|c|c|}
\hline & & $\begin{array}{l}\text { therapy evaluation, } 43 \% \text { with } \\
\text { splints, } 50 \% \text { with shoe lifts }\end{array}$ & & & & \\
\hline \multirow[t]{3}{*}{$\begin{array}{l}\text { Sornay-Soares } \\
\text { et al., [44] }\end{array}$} & \multirow[t]{3}{*}{ OBS } & $\begin{array}{l}\text { Children meeting the } 1997 \\
\text { Durban criteria for JIA with } \\
\text { knee involvement. }\end{array}$ & \multirow[t]{3}{*}{ 8/8 (13 knees) } & \multirow[t]{3}{*}{6 months, 12 months } & $\begin{array}{l}\text { Joint lavage using } 2 \text { needles and } \\
0.5-1.51 \mathrm{ml} \text { saline. Followed by } \\
\text { one vial of TH, except in } 2 \text { knees }\end{array}$ & \multirow[t]{3}{*}{$\begin{array}{l}\text { Sustained Clinical } \\
\text { Response: Pain } \\
\text { and joint effusion }\end{array}$} \\
\hline & & \multirow{2}{*}{$\begin{array}{l}\text { Co-interventions: } 6 / 8 \text { on NSAIDs, } \\
5 / 8 \text { on MTX, } 1 / 8 \text { on Azathioprine, } \\
2 / 8 \text { on Cyclosporine }\end{array}$} & & & & \\
\hline & & & & & $\begin{array}{l}\text { Post-injection: Knees were } \\
\text { taped and advised to rest for } \\
24 \text { hrs, keeping in extension } \\
\text { with walking crutches. Ice packs } \\
\text { could be used for pain relief. }\end{array}$ & \\
\hline \multirow[t]{4}{*}{$\begin{array}{l}\text { Verma } \\
\text { et al., [45] }\end{array}$} & \multirow[t]{4}{*}{ OBS } & $\begin{array}{l}\text { Diagnosis of unresponsive } \\
\text { oligoarticular/polyarticular JIA. }\end{array}$ & $\begin{array}{l}\text { 13/13 (13 knees and } 3 \\
\text { ankles) } 3 \text { patients were } \\
\text { lost to follow-up at } 6 \\
\text { months due to } \\
\text { uncontrolled arthritis. }\end{array}$ & \multirow[t]{4}{*}{6,12 weeks. } & TA (0.5-1 ml, 20-40 mg). & \multirow[t]{4}{*}{ Mid-leg circumference $(\mathrm{cm})$} \\
\hline & & $\begin{array}{l}\text { Joint swelling/effusion, limitation } \\
\text { of ROM, tenderness, pain, warmth. }\end{array}$ & \multirow[t]{3}{*}{$\begin{array}{l}4 \text { children were lost to } \\
\text { follow-up at } 12 \text { months. }\end{array}$} & & \multirow[t]{3}{*}{$\begin{array}{l}\text { Post-injection: Reduced } \\
\text { movement for } 24 \text { hrs. }\end{array}$} & \\
\hline & & $\begin{array}{l}12 \text { weeks daily oral naproxen } \\
\text { and/or weekly MTX. }\end{array}$ & & & & \\
\hline & & $\begin{array}{l}\text { Co-interventions: All patients } \\
\text { were on NSAIDs. }\end{array}$ & & & & \\
\hline \multirow[t]{7}{*}{$\begin{array}{l}\text { Zulian } \\
\text { et al., [46] }\end{array}$} & \multirow[t]{7}{*}{ OBS } & $\begin{array}{l}\text { Diagnosis persistent or } \\
\text { extended oligoarticular JIA }\end{array}$ & \multirow[t]{7}{*}{$\begin{array}{l}85 / 85 \text { ( } 115 \text { knees } \\
\text { and } 15 \text { ankles) }\end{array}$} & \multirow[t]{7}{*}{$\begin{array}{l}1,3,6,9,12,18 \\
\text { and } 24 \text { months }\end{array}$} & $\begin{array}{l}\text { TH: } 42 \text { patients treated; } \\
1 \mathrm{mg} / \mathrm{kg}(>40 \mathrm{mg})\end{array}$ & \multirow{7}{*}{$\begin{array}{l}\text { Sustained Clinical } \\
\text { Response: Swelling } \\
\text { and limited ROM } \\
\text { and pain and warmth. }\end{array}$} \\
\hline & & $\begin{array}{l}\text { Patients managed at University } \\
\text { of Padua paediatric } \\
\text { rheumatology unit }\end{array}$ & & & \multirow{3}{*}{$\begin{array}{l}\text { TA:43 patients treated; } \\
1 \mathrm{mg} / \mathrm{kg} \text { ( }>40 \mathrm{mg} \text { ) } \\
\text { (Availability issues } \\
\text { of TH meant TA was } \\
\text { used as an alternative } \\
\text { in some cases). }\end{array}$} & \\
\hline & & $\begin{array}{l}\text { Received IACls from Jan } \\
1996 \text { and Dec } 2000 .\end{array}$ & & & & \\
\hline & & $\begin{array}{l}\text { Unsatisfactory response } \\
\text { to NSAIDs. }\end{array}$ & & & & \\
\hline & & $\begin{array}{l}\text { Persistent isolated } \\
\text { joint involvement. }\end{array}$ & & & \multirow{3}{*}{$\begin{array}{l}\text { Post-injection: Non- } \\
\text { weight bearing for } \\
\text { at last } 72 \text { hrs post-injection. }\end{array}$} & \\
\hline & & $\begin{array}{l}\text { Co-interventions: TH: } 64.3 \% \\
\text { on NSAIDs, } 11.4 \% \text { on MTX }\end{array}$ & & & & \\
\hline & & $\begin{array}{l}\text { TA: } 51.7 \% \text { on NSAIDs, } \\
5 \% \text { on MTX }\end{array}$ & & & & \\
\hline
\end{tabular}

$\mathrm{OB}=$ observational; $\mathrm{RCT}=$ randomised controlled trial; $\mathrm{JA}=$ juvenile idiopathic arthritis; JCA = juvenile chronic arthritis; JRA $=$ juvenile rheumatoid arthritis; WHO $=$ World Health Organization; $\mathrm{ACR}=\mathrm{American}$ College of Rheumatology; EULAR = European League Against Rheumatism; ILR = International League of Associations for Rheumatology; ROM= range of motion; MTX=methotrexate; IACI(s) = intra-articular corticosteroid injection; $\mathrm{TH}=$ triamcinolone hexacetonide; $\mathrm{TA}=$ triamcinolone acetonide; $\mathrm{NSAID}(\mathrm{s})=$ Non-steroidal Anti-Inflammatory Drug(s); $\mathrm{MA}=$ methylprednisolone acetonide; $\mathrm{BM}=$ betamethasone; IACI(s) = intra-articular corticosteroid injection(s); US = ultrasound; MRI = magnetic imaging resonance; STJ = subtalar joint; MTPJ = metatarsophalangeal joint; IPJ = interphalangeal joint; SD = standard deviation; TNF-alpha = tumour necrosis factor alpha; DMARD = Disease Modifying Anti-Rheumatic Drug. 
Table 3 Quality assessment of included studies

\begin{tabular}{|c|c|c|c|c|c|c|c|c|}
\hline Author & $\begin{array}{l}\text { Sequence generation/ } \\
\text { allocation concealment } \\
\text { (internal validity) }\end{array}$ & $\begin{array}{l}\text { Blinding of participants, } \\
\text { personnel and outcome } \\
\text { assessors (internal validity) }\end{array}$ & $\begin{array}{l}\text { Incomplete outcome } \\
\text { data (internal validity) }\end{array}$ & $\begin{array}{l}\text { Selective outcome } \\
\text { reporting/statistical } \\
\text { issues (internal validity) }\end{array}$ & $\begin{array}{l}\text { Interventions } \\
\text { (internal validity) }\end{array}$ & $\begin{array}{l}\text { Generalisability } \\
\text { (external validity) }\end{array}$ & $\begin{array}{l}\text { Internal } \\
\text { validity }\end{array}$ & $\begin{array}{l}\text { External } \\
\text { validity }\end{array}$ \\
\hline Balogh et al., [30] & Low & Low & Low & High & Low & Low & Low & Low \\
\hline Al-wahadneh, [31] & $\mathrm{N} / \mathrm{A}$ & N/A & Low & Low & High & Low & Low & Low \\
\hline Allen et al., [32] & $\mathrm{N} / \mathrm{A}$ & N/A & Low & Low & Low & High & Low & High \\
\hline $\begin{array}{l}\text { Beukelman } \\
\text { et al., [26] }\end{array}$ & N/A & N/A & Low & Low & High & High & Low & High \\
\hline Cahill et al., [25] & N/A & N/A & Low & Low & Low & Low & Low & Low \\
\hline Earley et al., [33] & N/A & N/A & Low & Low & High & Low & Low & Low \\
\hline Eberhard et al., [34] & N/A & N/A & Low & Low & High & High & Low & High \\
\hline Eich et al., [35] & N/A & N/A & High & Low & Low & High & Low & High \\
\hline $\begin{array}{l}\text { Hertzberger-ten } \\
\text { Cate et al., [36] }\end{array}$ & N/A & N/A & Low & Low & High & Low & Low & Low \\
\hline Honkanen et al., [37] & N/A & N/A & Low & Low & High & Low & Low & Low \\
\hline Huppertz et al., [38] & N/A & N/A & High & Low & Low & Low & Low & Low \\
\hline Laurell et al., [24] & N/A & N/A & Low & Low & Low & High & Low & High \\
\hline Lepore et al., [39] & N/A & N/A & Low & Low & Low & Low & Low & Low \\
\hline Marti et al., [19] & $\mathrm{N} / \mathrm{A}$ & N/A & Low & Low & Low & High & Low & High \\
\hline $\begin{array}{l}\text { Papadopoulou } \\
\text { et al., [40] }\end{array}$ & $\mathrm{N} / \mathrm{A}$ & N/A & Low & High & High & High & Low & High \\
\hline Ravelli et al., [41] & $\mathrm{N} / \mathrm{A}$ & N/A & Low & Low & Low & High & Low & High \\
\hline Remedios et al., [42] & $\mathrm{N} / \mathrm{A}$ & N/A & Low & Low & Low & Low & Low & Low \\
\hline Sherry et al., [43] & $\mathrm{N} / \mathrm{A}$ & N/A & Low & High & High & Low & Low & Low \\
\hline $\begin{array}{l}\text { Sornay-Soares } \\
\text { et al., [44] }\end{array}$ & $\mathrm{N} / \mathrm{A}$ & N/A & Low & Low & High & High & Low & High \\
\hline Verma et al., [45] & $\mathrm{N} / \mathrm{A}$ & N/A & Low & Low & Low & Low & Low & Low \\
\hline Zulian et al., [46] & N/A & N/A & High & High & High & Low & High & Low \\
\hline
\end{tabular}


Table 4 Qualitative synthesis of results and overview of evidence

\begin{tabular}{|c|c|c|c|c|c|}
\hline Domain & Author, year (ref.) & Results & Adverse effects & Level of evidence (specific areas) & Level of evidence (for domain) \\
\hline \multirow[t]{4}{*}{ Tenderness/pain } & Eich et al, [35] & $\begin{array}{l}\text { Knee: } 0 \% \text { after } 1 \text { month } \\
\text { (no significance value given) }\end{array}$ & $\begin{array}{l}\text { One patient experienced focal } \\
\text { cutaneous atrophy and a possible } \\
\text { ruptured popliteal cyst }\end{array}$ & $\begin{array}{l}\text { Weak evidence for IACls } \\
\text { decreasing pain in the } \\
\text { knee as } 2 \text { studies show } \\
\text { a reduction of pain }\end{array}$ & $\begin{array}{l}\text { Weak evidence for IACls } \\
\text { decreasing pain in lower } \\
\text { leg joints overall as } 3 \text { studies } \\
\text { show a reduction of pain }\end{array}$ \\
\hline & Huppertz et al, [38] & $\begin{array}{l}\text { Knee: } 33.3 \% \text { after } 7 \text { weeks } \\
\text { (no significance value given) }\end{array}$ & Not Specified & & \\
\hline & \multirow[t]{2}{*}{ Laurell et al, [24] } & $\begin{array}{l}\text { Ankle: Pain regression/partial } \\
\text { improvement }=92.5 \% \\
\text { (no specific data included, } \\
\text { only percentages) after } \\
4 \text { weeks }\end{array}$ & $\begin{array}{l}\text { Local subcutaneous atrophy } \\
\text { in } 3 \text { patients at } 4 \text { injection sites }\end{array}$ & \multirow[t]{2}{*}{ Inconclusive due to lack of studies } & \\
\hline & & (No significance value given) & & & \\
\hline \multirow[t]{2}{*}{ Swelling } & Eich et al, [35] & $\begin{array}{l}\text { Knee: } 27.3 \% \text { after } 1 \text { month } \\
\text { (no significance value given) }\end{array}$ & $\begin{array}{l}\text { One patient experienced focal } \\
\text { cutaneous atrophy and a possible } \\
\text { ruptured popliteal cyst }\end{array}$ & & $\begin{array}{l}\text { Weak evidence for IACls } \\
\text { decreasing swelling in lower } \\
\text { leg joints overall as } 2 \text { studies } \\
\text { show a reduction in swelling }\end{array}$ \\
\hline & Huppertz et al, [38] & $\begin{array}{l}\text { Knee: } 0 \% \text { after } 7 \text { weeks } \\
\text { (no significance value given) }\end{array}$ & Not Specified & & \\
\hline \multirow[t]{11}{*}{ Synovitis } & \multirow{5}{*}{$\begin{array}{l}\text { Papadopoulou et al., } \\
{[40]}\end{array}$} & Number of joints in remission vs. & \multirow{2}{*}{$\begin{array}{l}0.9 \% \text { of injected joints suffered } \\
\text { from skin hypopigmentation } \\
\text { or subcutaneous atrophy. }\end{array}$} & \multirow{3}{*}{$\begin{array}{l}\text { Weak evidence for IACls decreasing } \\
\text { synovitis in the knee, ankle and } \\
\text { STJ as } 2 \text { studies for each joint } \\
\text { showed a reduction of synovitis. }\end{array}$} & \multirow{5}{*}{$\begin{array}{l}\text { Weak evidence for IACIs } \\
\text { decreasing synovitis in lower } \\
\text { leg joints overall as } 3 \text { studies } \\
\text { show reduction of synovitis }\end{array}$} \\
\hline & & $\begin{array}{l}\text { Number of joints with synovitis } \\
\text { flare: Knee }=7920 \text { vs. } 208 \%\end{array}$ & & & \\
\hline & & $(p<0.001) ;$ Ankle $=54.8 \%$ vs. & \multirow{3}{*}{$\begin{array}{l}\text { "A few" patients developed } \\
\text { flushing or redness of the } \\
\text { cheeks } 24-48 \text { hrs post-injection. }\end{array}$} & & \\
\hline & & $\begin{array}{l}45.2 \%(p=0.14) \text { STJ }=65.5 \% \text { vs. } \\
34.5 \%(p<0.0001) ; M T P J=85.7 \% \\
\text { vs. } 14.2 \%(p=0.008) ; I P J=90.0 \% \\
\text { vs. } 10.0 \%(p=0.0003) .\end{array}$ & & $\begin{array}{l}\text { Inconclusive evidence for MTPJ } \\
\text { and IPJ due to lack of studies }\end{array}$ & \\
\hline & & $\begin{array}{l}\text { Overall mean relapse time } 0.5 \text { yrs } \\
\text { (IQR 0.3-1.3 yrs) vs. mean remission } \\
\text { time } 0.9 \text { yrs (IQR 0.6-1.9 yrs). }\end{array}$ & & & \\
\hline & \multirow[t]{3}{*}{ Ravelli et al., [41] } & $\begin{array}{l}\text { Knee: continued resolution } \\
\text { at } 6 \text { months }=69 \%\end{array}$ & \multirow{3}{*}{$\begin{array}{l}\text { One patient suffered from } \\
\text { subcutaneous atrophy at } \\
\text { the injection site. }\end{array}$} & & \\
\hline & & Reoccurrence/relapse $=31 \%$ & & & \\
\hline & & (no significance value given) & & & \\
\hline & \multirow[t]{3}{*}{ Remedios et al., [42] } & Clinical synovitis vs. MRI pannus: & \multirow[t]{3}{*}{ Not Specified. } & & \\
\hline & & $\begin{array}{l}\text { Tibiotalar: } 84.6 \% \text { vs. } 84.6 \% ; \text { STJ: } \\
23.1 \% \text { (definite) vs. } 46.2 \% \\
\text { (no significance value given) }\end{array}$ & & & \\
\hline & & $\begin{array}{l}\text { Mean duration of effect (guided } \\
\text { vs. unguided) (weeks): } 38.3 \text { vs. } \\
13.9 \text { (no significance value given) }\end{array}$ & & & \\
\hline Effusion & Huppertz et al, [38] & $\begin{array}{l}\text { Knee: } 13.3 \% \text { after } 7 \text { weeks } \\
\text { (no significance value given) }\end{array}$ & Not Specified & & $\begin{array}{l}\text { Inconclusive due to lack } \\
\text { of studies }\end{array}$ \\
\hline
\end{tabular}

$0 \%$ after 7 weeks

Not Specified skin hypopigmentation

"A few" patients developed

"A ushing or redness of the

v. $10.0 \%(p=0.0003)$

Overall mean relapse time 0.5 yrs

One patient suffered from

subcutaneous atrophy at

Reoccurrence/relapse $=31 \%$

Knee: $13.3 \%$ after 7 weeks 
Table 4 Qualitative synthesis of results and overview of evidence (Continued)

\begin{tabular}{|c|c|c|c|}
\hline Hyperthermia & Eich et al, [35] & $\begin{array}{l}\text { Knee: } 18.2 \% \text { after } 1 \text { month } \\
\text { (no significance value given) }\end{array}$ & $\begin{array}{l}\text { One patient experienced focal } \\
\text { cutaneous atrophy and a } \\
\text { possible ruptured popliteal cyst }\end{array}$ \\
\hline \multirow[t]{12}{*}{$\begin{array}{l}\text { Sustained clinical } \\
\text { response }\end{array}$} & Al-Wahadneh, [31] & $\begin{array}{l}\text { Knee: } 30 / 30=100 \% \text { maintained } \\
\text { resolution at } 3 \text { months } \\
\text { (no significance value given) }\end{array}$ & $\begin{array}{l}\text { 1/24 (4\%): short-lived pain and } \\
\text { erythema, } 2 / 24 \text { (8\%): subcutaneous } \\
\text { atrophy resolved dramatically after } \\
\text { one year, } 2 / 24 \text { (8\%): asymptomatic } \\
\text { periarticular calcification. }\end{array}$ \\
\hline & Allen et al, [35] & $\begin{array}{l}\text { Knee: } 18 / 48=37.5 \% \text { relapse at } \\
6 \text { months (no significance } \\
\text { value given) }\end{array}$ & $\begin{array}{l}\text { Subcutaneous fat atrophy at } \\
\text { injection site in one patient. }\end{array}$ \\
\hline & \multirow[t]{2}{*}{ Beukelman et al, [26] } & $\begin{array}{l}\text { Knee: } 1.4 \text { yrs of resolution } \\
\text { before relapse }(S D \pm 1.0)\end{array}$ & \multirow{2}{*}{$\begin{array}{l}\text { 53\%: subcutaneous atrophy } \\
\text { or hypopigmentation at } \\
\text { injection site. }\end{array}$} \\
\hline & & $\begin{array}{l}\text { STJ: } 1.2 \text { yrs of resolution before } \\
\text { relapse }(T H+T A)(S D \pm 0.9)\end{array}$ & \\
\hline & \multirow[t]{2}{*}{ Earley et al, [33] } & $\begin{array}{l}\text { Knee: Excellent/Good Outcome: } \\
92.8 \% \text { vs. }\end{array}$ & \multirow[t]{2}{*}{$\begin{array}{l}2 / 23 \text { had areas of subcutaneous } \\
\text { atrophy at injection site. }\end{array}$} \\
\hline & & $\begin{array}{l}\text { Poor/Re-injected: } 7.2 \% \text { at } 3 \text { months } \\
\text { (No significance value given) }\end{array}$ & \\
\hline & \multirow[t]{4}{*}{ Eberhard et al, [34] } & Knee Median Relapse (months) & \multirow[t]{4}{*}{ Not Specified. } \\
\hline & & TH: $11.1+/-0.81$ & \\
\hline & & TA: $7.95+/-0.95$ & \\
\hline & & $(p=0.0072)$ & \\
\hline & \multirow[t]{2}{*}{$\begin{array}{l}\text { Hertzberger-ten } \\
\text { Cate et al, [36] }\end{array}$} & \multirow{2}{*}{$\begin{array}{l}\text { Knee: resolution maintained in } \\
70 \% \text { of knees for }>6 \text { months } \\
\text { (No significance value given) }\end{array}$} & $\begin{array}{l}\text { 2/21 patients suffered a small } \\
\text { atrophic lesion at the injection site. }\end{array}$ \\
\hline & & & $\begin{array}{l}1 / 21 \text { patient suffered a red and } \\
\text { painful knee the day following } \\
\text { injection (resolved by local } \\
\text { ice application). }\end{array}$ \\
\hline \multirow[t]{3}{*}{$\begin{array}{l}\text { Sustained clinical } \\
\text { response (cont'd) }\end{array}$} & Honkanen et al, [37] & $\begin{array}{l}\text { Knee: overall probability of } \\
\text { sustained clinical response } \\
\text { was higher for TH then MP } \\
\text { after } 6-8 \text { weeks }(p>0.0005)\end{array}$ & Not Specified. \\
\hline & Laurell et al, [24] & $\begin{array}{l}\text { Ankle pain Regression/Partial } \\
\text { Improvement }=92.5 \% \text { (no } \\
\text { specific data included, only } \\
\text { percentages) after } 4 \text { weeks } \\
\text { (No significance value given) }\end{array}$ & $\begin{array}{l}\text { Local subcutaneous atrophy } \\
\text { in } 3 \text { patients at } 4 \text { injection sites. }\end{array}$ \\
\hline & Lepore et al, [39] & $\begin{array}{l}\text { Knee: } \text { mean remission } \\
\text { time }=13.9 \text { months }\end{array}$ & $\begin{array}{l}10 \% \text { ( } 3 \text { patients) subcutaneous } \\
\text { lipolysis with spontaneous } \\
\text { regression. }\end{array}$ \\
\hline
\end{tabular}

Inconclusive due to lack of studies

Weak evidence for IACls decreasing Weak evidence for IACls knee, ankle and STJ as 11,4 and 2 studies respectively showed and symptoms in lower leg sustained clinical response. joints overall as 21 st show sustained clinical Inconclusive evidence for response 
Table 4 Qualitative synthesis of results and overview of evidence (Continued)

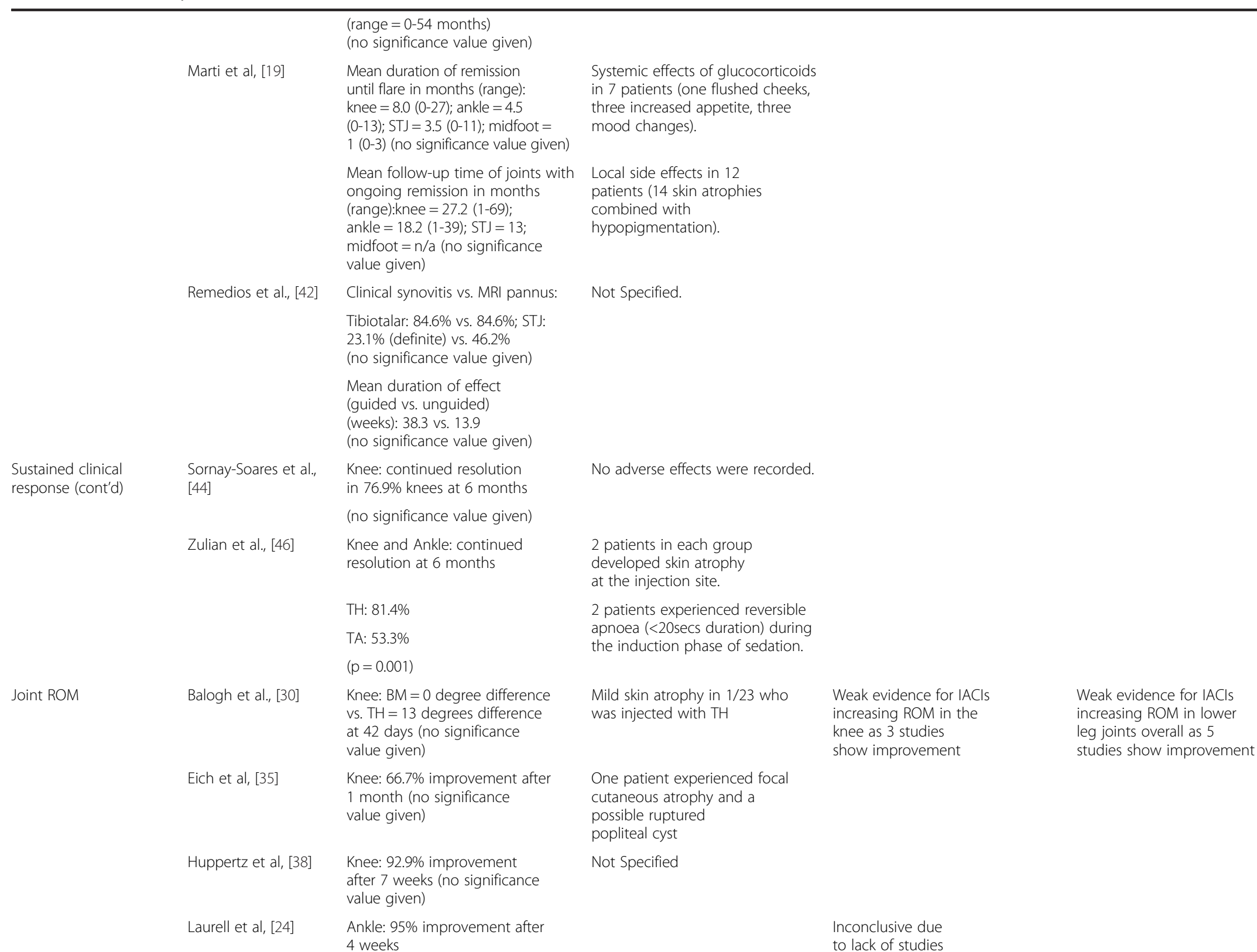


Table 4 Qualitative synthesis of results and overview of evidence (Continued)

\begin{tabular}{|c|c|c|c|c|c|}
\hline & & (No significance value given) & $\begin{array}{l}\text { Local subcutaneous } \\
\text { atrophy in } 3 \text { patients } \\
\text { at } 4 \text { injection sites }\end{array}$ & & \\
\hline & Cahill et al., [25] & $\begin{array}{l}\text { STJ: } 89.5 \% \text { returned to normal } \\
\text { ROM within } 13 \text { weeks } \\
\text { (no significance value given). } \\
\text { Mean duration of improvement = } \\
1.2 \text { SD } \pm 0.9 \text { yrs. }\end{array}$ & $\begin{array}{l}\text { Subcutaneous atrophy or } \\
\text { hypopigmentation in 53\% }\end{array}$ & $\begin{array}{l}\text { Inconclusive due } \\
\text { to lack of studies }\end{array}$ & \\
\hline \multirow[t]{5}{*}{$\begin{array}{l}\text { Leg length } \\
\text { discrepancy }\end{array}$} & Eich et al, [35] & $\begin{array}{l}\text { Improvement after } 1 \text { month: } \\
100 \% \text { (no significance } \\
\text { value given) }\end{array}$ & $\begin{array}{l}\text { One patient experienced } \\
\text { focal cutaneous atrophy } \\
\text { and a possible ruptured } \\
\text { popliteal cyst }\end{array}$ & & $\begin{array}{l}\text { Weak evidence for IACls } \\
\text { decreasing LLD as } 3 \\
\text { studies show reduction }\end{array}$ \\
\hline & Sherry et al., [43] & $\begin{array}{l}\text { Mean difference in leg length } \\
\text { for early intervention vs. control: }\end{array}$ & Not Specified & & \\
\hline & & $0 \%( \pm 0)$ vs. $1.0 \%( \pm 1.4)(p=0.005)$ & & & \\
\hline & & $\begin{array}{l}\text { over mean follow up of } 4 \text { months } \\
\text { ( } S D \pm 11 \text { months) }\end{array}$ & & & \\
\hline & Verma et al., [45] & $\begin{array}{l}\text { Mean Lower Leg Difference } \\
(\mathrm{cm}): \downarrow 0.22 \text { at } 6 \text { and } 12 \\
\text { weeks (no significance } \\
\text { value given). }\end{array}$ & $\begin{array}{l}\text { No adverse effects } \\
\text { were recorded. }\end{array}$ & & \\
\hline \multirow[t]{3}{*}{ Circumference } & Balogh et al., [30] & $\begin{array}{l}\text { Mean Knee Joint } \\
\text { Circumference }(\mathrm{cm}) \text { : }\end{array}$ & $\begin{array}{l}\text { Mild skin atrophy in } 1 / 23 \\
\text { who was injected with TH }\end{array}$ & & $\begin{array}{l}\text { Inconclusive due to } \\
\text { lack of studies }\end{array}$ \\
\hline & & $\mathrm{BM}=1.0$ & & & \\
\hline & & $\begin{array}{l}\text { TH }=-1.7 \text { after } 1,3,7 \text { and } \\
42 \text { days (no significance } \\
\text { values given). }\end{array}$ & & & \\
\hline Imaging & & & & & $\begin{array}{l}\text { Weak evidence for IACIs decreasing } \\
\text { imaging findings in lower leg joints } \\
\text { overall as } 3 \text { studies show improvement }\end{array}$ \\
\hline MR imaging & & & & & $\begin{array}{l}\text { Weak evidence for IACIs decreasing } \\
\text { MRI detectable clinical signs and } \\
\text { symptoms as } 3 \text { studies show } \\
\text { detectable improvement }\end{array}$ \\
\hline \multirow[t]{2}{*}{ Effusion } & Eich et al., [35] & $\begin{array}{l}\text { Knee: } 36.4 \% \text { detected by } \\
\text { MRI at } 1 \text { week and } 1 \text { month } \\
\text { (no significance value given) }\end{array}$ & $\begin{array}{l}\text { One patient experienced } \\
\text { focal cutaneous atrophy } \\
\text { and a possible ruptured } \\
\text { popliteal cyst }\end{array}$ & $\begin{array}{l}\text { Weak evidence for IACls } \\
\text { decreasing MRI detectable } \\
\text { effusion as } 2 \text { studies show } \\
\text { detectable improvement. }\end{array}$ & \\
\hline & Huppertz et al, [38] & $\begin{array}{l}\text { Knee and Ankle: } 40.0 \% \text { at } \\
7 \text { and } 13 \text { weeks (no } \\
\text { significance value given) }\end{array}$ & Not Specified. & & \\
\hline Pannus & Eich et al., [35] & $\begin{array}{l}\text { Knee: } 63.6 \% \text { detected by } \\
\text { MRI at } 1 \text { week and } 1 \text { month } \\
\text { (no significance value given) }\end{array}$ & $\begin{array}{l}\text { One patient experienced } \\
\text { focal cutaneous atrophy }\end{array}$ & $\begin{array}{l}\text { Weak evidence for IACls } \\
\text { decreasing MRI detectable }\end{array}$ & \\
\hline
\end{tabular}


Table 4 Qualitative synthesis of results and overview of evidence (Continued)

\begin{tabular}{|c|c|c|c|c|c|}
\hline & & & $\begin{array}{l}\text { and a possible ruptured } \\
\text { popliteal cyst }\end{array}$ & $\begin{array}{l}\text { pannus as } 3 \text { studies show } \\
\text { detectable improvement. }\end{array}$ & \\
\hline & Huppertz et al, [38] & $\begin{array}{l}\text { Knee and Ankle: } 10.0 \% \text { at } 7 \\
\text { and } 13 \text { weeks (no significance } \\
\text { value given) }\end{array}$ & Not Specified. & & \\
\hline & Remedios et al., [42] & Clinical synovitis vs. MRI pannus: & Not Specified. & & \\
\hline & & $\begin{array}{l}\text { Tibiotalar: } 84.6 \% \text { vs. } 84.6 \% \text {; STJ: } \\
23.1 \% \text { (definite) vs. } 46.2 \% \\
\text { (no significance value given) }\end{array}$ & & & \\
\hline & & $\begin{array}{l}\text { Mean duration of effect } \\
\text { (guided vs. unguided) } \\
\text { (weeks): } 38.3 \text { vs. } 13.9 \\
\text { (no significance value given) }\end{array}$ & & & \\
\hline Popliteal cyst & Eich et al., [35] & $\begin{array}{l}\text { Knee: } 33.3 \% \text { detectable } \\
\text { at } 1 \text { week and } 1 \text { month } \\
\text { (no significance value given) }\end{array}$ & $\begin{array}{l}\text { One patient experienced } \\
\text { focal cutaneous atrophy } \\
\text { and a possible ruptured } \\
\text { popliteal cyst }\end{array}$ & $\begin{array}{l}\text { Inconclusive due to } \\
\text { lack of studies }\end{array}$ & \\
\hline $\begin{array}{l}\text { Destruction of articular } \\
\text { cartilage/bone }\end{array}$ & Eich et al., [35] & $\begin{array}{l}\text { Missing outcome data at } \\
1 \text { week and } 1 \text { month } \\
\text { (no significance value given) }\end{array}$ & $\begin{array}{l}\text { One patient experienced } \\
\text { focal cutaneous atrophy } \\
\text { and a possible ruptured } \\
\text { popliteal cyst }\end{array}$ & $\begin{array}{l}\text { Inconclusive due to } \\
\text { lack of studies }\end{array}$ & \\
\hline Destruction of meniscus & Eich et al., [35] & $\begin{array}{l}\text { 100\% (no ligament } \\
\text { destruction reported) } \\
\text { at } 1 \text { week and } 1 \text { month } \\
\text { (no significance value given) }\end{array}$ & $\begin{array}{l}\text { One patient experienced } \\
\text { focal cutaneous atrophy } \\
\text { and a possible ruptured } \\
\text { popliteal cyst }\end{array}$ & $\begin{array}{l}\text { Inconclusive due to } \\
\text { lack of studies }\end{array}$ & \\
\hline Bone marrow oedema & Eich et al., [35] & $\begin{array}{l}\text { None reported at } 1 \\
\text { week and } 1 \text { month } \\
\text { (no significance value given) }\end{array}$ & $\begin{array}{l}\text { One patient experienced } \\
\text { focal cutaneous atrophy } \\
\text { and a possible ruptured } \\
\text { popliteal cyst }\end{array}$ & $\begin{array}{l}\text { Inconclusive due to } \\
\text { lack of studies }\end{array}$ & \\
\hline Avascular necrosis & Eich et al., [35] & $\begin{array}{l}\text { None reported at } 1 \\
\text { week and } 1 \text { month } \\
\text { (no significance value given) }\end{array}$ & $\begin{array}{l}\text { One patient experienced } \\
\text { focal cutaneous atrophy } \\
\text { and a possible ruptured } \\
\text { popliteal cyst }\end{array}$ & $\begin{array}{l}\text { Inconclusive due to } \\
\text { lack of studies }\end{array}$ & \\
\hline $\begin{array}{l}\text { Uptake of contrast } \\
\text { medium }\end{array}$ & Huppertz et al, [38] & $\begin{array}{l}\text { Knee and Ankle: } 20.0 \% \\
\text { detectable at } 7 \text { and } 13 \\
\text { weeks (no significance } \\
\text { value given) }\end{array}$ & Not Specified. & $\begin{array}{l}\text { Inconclusive due to } \\
\text { lack of studies }\end{array}$ & \\
\hline US imaging & & & & & $\begin{array}{l}\text { Weak evidence for IACls } \\
\text { decreasing US detectable } \\
\text { clinical signs and symptoms } \\
\text { as } 2 \text { studies show } \\
\text { detectable improvement }\end{array}$ \\
\hline $\begin{array}{l}\text { Joint effusion } \\
\text { and/or pannus }\end{array}$ & Eich et al., [35] & $\begin{array}{l}\text { Knee: } 100 \% \text { detectable after } \\
1 \text { week and } 1 \text { month } \\
\text { (no significance value given) }\end{array}$ & $\begin{array}{l}\text { One patient experienced } \\
\text { focal cutaneous atrophy }\end{array}$ & $\begin{array}{l}\text { Inconclusive due to } \\
\text { lack of studies }\end{array}$ & \\
\hline
\end{tabular}

Knee: $33.3 \%$ detectable (no significance value given)

One patient experienced utaneous atrophy (apliteal cyst destruction reported) 1 week and 1 month

None reported at 1 week and 1 month

None reported at 1

Knee and Ankle: 20.0\%

ctable at 7 and 13

weeks (no significance

value given)

Weak eving US detectable

clinical signs and symptoms

as 2 studies show 
Table 4 Qualitative synthesis of results and overview of evidence (Continued)

\begin{tabular}{|c|c|c|c|c|}
\hline & & & $\begin{array}{l}\text { and a possible ruptured } \\
\text { popliteal cyst }\end{array}$ & \\
\hline Popliteal cyst & Eich et al., [35] & $\begin{array}{l}\text { Knee: } 0 \% \text { detectable after } 1 \\
\text { week and } 1 \text { month } \\
\text { (no significance value given) }\end{array}$ & $\begin{array}{l}\text { One patient experienced } \\
\text { focal cutaneous atrophy } \\
\text { and a possible ruptured } \\
\text { popliteal cyst }\end{array}$ & $\begin{array}{l}\text { Inconclusive due to } \\
\text { lack of studies }\end{array}$ \\
\hline \multirow[t]{4}{*}{ Synovial hypertrophy } & \multirow[t]{4}{*}{ Laurell et al., [24] } & $\begin{array}{l}\text { Talocrural joint }=87 \% \\
\text { regression vs. } 13 \% \text { no effect }\end{array}$ & \multirow{4}{*}{$\begin{array}{l}\text { Local subcutaneous } \\
\text { atrophy in } 3 \text { patients } \\
\text { at } 4 \text { injection sites. }\end{array}$} & \multirow[t]{4}{*}{$\begin{array}{l}\text { Inconclusive due to } \\
\text { lack of studies }\end{array}$} \\
\hline & & $\begin{array}{l}\text { Posterior-STJ = 95\% regression } \\
\text { vs. } 5 \% \text { no effect }\end{array}$ & & \\
\hline & & $\begin{array}{l}\text { Midfoot joints }=91 \% \\
\text { regression vs. 9\% no effect } \\
\text { (no significance value given). }\end{array}$ & & \\
\hline & & $\begin{array}{l}\text { Mean synovial thickness: } \\
\text { statistically significant } \\
\text { difference at } 4 \text { weeks } \\
(p<0.001)\end{array}$ & & \\
\hline \multirow[t]{3}{*}{ Synovial hyperaemia } & \multirow[t]{3}{*}{ Laurell et al., [24] } & $\begin{array}{l}\text { Talocrural Joint }=86 \% \\
\text { normalisation vs. } 14 \% \\
\text { no normalisation }\end{array}$ & \multirow[t]{3}{*}{$\begin{array}{l}\text { Local subcutaneous } \\
\text { atrophy in } 3 \text { patients } \\
\text { at } 4 \text { injection sites. }\end{array}$} & \multirow[t]{3}{*}{$\begin{array}{l}\text { Inconclusive due to } \\
\text { lack of studies }\end{array}$} \\
\hline & & $\begin{array}{l}\text { Posterior-STJ }=95 \% \\
\text { normalisation vs. } 5 \% \text { no } \\
\text { normalisation }\end{array}$ & & \\
\hline & & $\begin{array}{l}\text { Midfoot Joints }=80 \% \\
\text { normalisation vs. } 20 \% \\
\text { no normalisation } \\
\text { (no significance value } \\
\text { given) after } 4 \text { weeks. }\end{array}$ & & \\
\hline
\end{tabular}

ssible ruptured

One patient experienced

conclusive due to

Inconclusive due to

Abbreviations: STJ subtalar joint, SD standard deviation, IACI/S) intra articular corticosteroid injection(s), $T H$ triam,inolone hexacetonide, $T A$ triamcinolone acetonide, MP methylprednisolone acetate,

$B M$ betamethasone, MRI magnetic resonance imaging US ultrasound, IQR inter-quartile range, ROM range of motion, LLD leg length discrepancy, MTPJ metatarsophalangeal joint, IPJ interphalangeal joint. 


\section{Tenderness/pain}

Three observational studies investigated measures of tenderness/pain in the lower leg joints, inclusive of the knee and ankle $[24,35,38]$. While all studies showed a reduction in pain after utilising IACIs, no significance values were given $[24,35,38]$. A weak level of evidence was found for IACIs decreasing lower leg tenderness/pain. Weak evidence for IACIs decreasing tenderness/pain was also found specifically in the knee (2 studies) and inconclusive evidence in the ankle due to the lack of studies (1 study).

\section{Swelling}

Two observational studies investigated a reduction of swelling in the knee $[35,38]$. While no significance values were reported, there was weak evidence of IACIs decreasing swelling.

\section{Synovitis}

Three observational studies investigated a reduction of synovitis in lower limb joints inclusive of the knee ankle, STJ, MTPJ and IPJ [40-42]. Inter-quartile ranges (IQR) were only available from one study reporting the overall mean relapse time of 0.5 yrs (IQR 0.3-1.3 yrs) compared with the mean remission time of 0.9 yrs (IQR 0.6-1.9 yrs) [40]. No significance values were given for the other studies $[41,42]$. A weak level of evidence was found for IACIs decreasing synovitis in lower leg joints. Specifically, a weak level of evidence was found for decreasing synovitis in the knee, ankle and STJ as two studies showed a reduction. There was inconclusive evidence for all other lower limb joints including MTPJ and IPJ.

\section{Effusion}

Only one observational study investigated a reduction of effusion in the knee [38] and no significance values were given. There was inconclusive evidence for IACIs decreasing synovitis in the knee due to lack of studies.

\section{Hyperthermia}

Only one observational study investigated a reduction of hyperthermia in the knee [35] and no significance values were given. There was inconclusive evidence for IACIs decreasing hyperthermia in the knee due to lack of studies.

\section{Sustained clinical response}

Thirteen observational studies investigated a sustained clinical response post-injection in the lower leg joints, inclusive of the knee, ankle and foot [19,24,26,31-34, $36,37,39,42,44,46]$. Sustained clinical response included measures of pain, oedema, erythema and general inflammation, and was determined over periods ranging from 166 months post-injection, depending on joints investigated. Four studies showed statistically significant sustained clinical response $[26,34,37,46]$. Nine studies also found sustained clinical response in relation to duration of improvement and reduction/resolution of pain, inflammation, limitation of ROM, joint tenderness, swelling, temperature, joint effusion, deformity, synovitis, contracture and gait abnormalities. However, these results were derived from descriptive statistical analyses only [19,24,31-33,36,39,41,44]. Two studies found statistically significant sustained clinical response in the knee [34,37]. Six studies also found sustained clinical response in the knee. However, no significance values were given as data was presented using descriptive statistics only $[31-33,36,39,44]$. One study found statistical significance for the knee and ankle collectively [46]. Sustained clinical response was also found in three studies for the ankle $[19,24,42]$, three studies for the STJ $[19,26,42]$, and one study for the midfoot [19]. However, no significance values were given. Overall, there was weak evidence for IACIs decreasing clinical signs and symptoms in lower leg joints as thirteen studies showed a sustained clinical response post-injection.

\section{Joint ranges of motion}

One RCT and four observational studies investigated joint ROM post-injection. The efficacy of IACIs was investigated for the STJ [25], knee [30,35,38] and ankle [24]. However, an odds ratio was only reported by one study for a mean duration of improvement of $1.2 \mathrm{yrs}$ (SD $\pm 0.9 \mathrm{yrs}$ ) [25]. Weak evidence was shown for IACIs increasing $\mathrm{ROM}$ in lower leg joints collectively. The RCT showed an increase in knee flexion following administrations of triamcinolone hexacetonide $(\mathrm{TH})$ and betamethasone (BM) [30]. TH increased knee flexion more than BM [43]. Two observational studies also showed improvement of knee ROM [35,38] and weak evidence was found for IACIs increasing ROM in the knee. One observational study showed increased foot inversion and eversion [25] and ankle joint ROM normalisation/partial improvement [24]. Overall, there was inconclusive evidence for IACIs improving joint ROM in the STJ and ankle.

\section{Leg length discrepancy}

One RCT and two observational studies investigated length discrepancies (LLD) [30,43,45]. Two studies specifically investigated LLD $[43,45]$. While both studies showed a decrease in the differences between limb length, only one was tested statistically and achieved statistical significance [43]. The other study only used descriptive statistics [45]. A weak level of evidence was found for decrease in lower LLD. One study also found a decrease in knee joint circumference; however, this was not statistically tested [30]. This particular study demonstrated that there was a decrease in knee joint circumference with use of $\mathrm{TH}$ or $\mathrm{BM}$ [30] but $\mathrm{TH}$ had a greater reduction compared to 
BM. Overall, there was inconclusive evidence for IACIs reducing knee circumference due to an insufficient number of studies.

\section{Disease activity outcomes detected by imaging}

Four observational studies investigated the ability of imaging techniques to detect the efficacy of IACIs on JIA disease activity [24,35,38,42]. These studies showed IACIs led to clinical effects on disease activity as detected by imaging techniques when compared to clinical assessment. However, these differences were not compared using inferential statistics. Overall, there was weak evidence for the use of imaging techniques detecting the effects of IACIs. Specifically, two studies investigated the effects of IACIs using MRI to effusion [35,38]. Three studies investigated the effects of IACIs using MRI to detect pannus [31,34,44]. There was weak evidence for IACIs decreasing MRI detectable effusion and pannus. One study investigated MRI for detecting the effects of IACIs reducing popliteal cysts, destruction of articular cartilage/bone, destruction of meniscus, bone marrow oedema and avascular necrosis [35]. One study investigated MRI for detecting the effects of IACIs reducing the uptake of contrast medium [38]. However, there was inconclusive evidence for these subdomains due to a lack of studies. Overall, the level of evidence was weak due to two studies showing the effects of IACIs using MRI.

Two studies investigated the effects of IACS with musculoskeletal ultrasound imaging [24,35]. One study found decreases in signs of joint effusion and/or pannus and popliteal cyst [24], and one study found decreases in signs of synovial hypertrophy and synovial hyperaemia [35]. Therefore, the level of evidence assigned was weak for IACIs decreasing ultrasound detectable signs and symptoms, and inconclusive evidence for each specific subdomain.

\section{Discussion}

Weak levels of evidence were found for IACIs decreasing tenderness/pain, swelling, synovitis, clinical signs and symptoms, increasing joint (ROM) in lower limb joints and medical imaging disease-related outcomes such as joint effusion, synovial hypertrophy and erosion. Inconclusive evidence was present for IACIs reducing effusion, hyperthermia, LLD and knee circumference. The majority of studies were low quality observational studies with one low quality RCT, and as such these conclusions are based upon weak evidence only. Additionally, the majority of the studies identified by the search strategy presented results using descriptive statistics only, leading to uncertainty regarding the effectiveness of IACIs versus a suitable comparator.

The results of this contemporary review demonstrate similar issues raised by previous reviews concerning the lack of conclusive evidence in support of IACIs improving clinical outcomes [16,17]. The adopted methodology of the most relevant previous systematic review appeared to be logical in restricting the inclusion criteria to RCTs in an attempt to increase the quality of evidence retrieved. However, at the time, there was a lack of published RCTs available for inclusion in this review and it subsequently concluded there was insufficient research to ascertain the effectiveness of IACIs for JIA. Observational studies were not considered by the authors as part of the inclusion criteria for that review, and as such potentially important studies were excluded which may have provided at least a weak level of evidence concerning IACI efficacy. In contrast, our review included observational studies and found weak evidence to suggest that sustained clinical response and improved joint ROM can be achieved following administration of IACIs to the joints of the lower limb in children with JIA.

A narrative literature review which adopted systematic search criteria on all medical treatment modalities for JIA including IACIs has previously been conducted [10]. The authors concluded there was significant evidence of efficacy for the use of IACIs in persistent oligoarticular JIA and moderate efficacy of IACIs for the use in polyarticular JIA based upon the findings of three studies [30,46,47]. However, this was not a true systematic review as the appraisal strategy did not adopt rigorous methods of quality assessment or evidence grading, and their conclusions were based solely upon effect sizes greater than $50 \%$ of patients. The lack of evidence grading introduces ambiguity to the conclusion of such reviews.

Our systematic review has utilised a specific quality assessment criteria with emphasis on the internal and external validity of the included studies [18]. Our study showed that while there was only one observational study with high internal validity [42], nine observational studies showed high external validity $[19,24,26,32,34,35,40,44,46]$. This may be explained by the fact that recruitment was largely conducted alongside standard practice within paediatric rheumatology outpatient clinics, thus increasing the clinical relevance of the results. This external validity criterion was also necessary to assess whether the study methodologies were pragmatic, as opposed to explanatory [48]. The pragmatic nature of these studies meant their conclusions were more likely to be applicable to the general population of children/adolescents with JIA to whom IACIs may be a possible treatment option.

Due to the lack of multiple RCTs in this area, no metaanalysis could be conducted. This has implications for future research as high quality RCTs and meta-analyses are necessary to inform evidence-based clinical practice. Indeed a pragmatic RCT of IACIs versus a suitable comparator for the relief of localised synovitis could have sufficient external validity to inform and influence current practice and health policy in future. However, it is recognised that several clinical practice guidelines recommend use of IACIs in spite of the lack of high quality research 
evidence supporting their use [10,20]. As such, it is acknowledged that a placebo- or sham-control arm for a parallel trial of IACIs may not be possible due to ethical restraints surrounding the withholding of standard care. A contextual problem with the lack of robust RCTs to evaluate IACIs at present exists with regards to recent changes to the Australian Medicare Benefits Schedule [49]. Evaluations of the cost-effectiveness of interventions are normally embedded within RCTs. At present, and to the best of the authors' knowledge, an evaluation of the costeffectiveness of IACIs in JIA has yet to be conducted. This raises an important issue; because presently people with arthritic conditions in Australia are potentially required to self-fund the cost of each individual IACI they receive [49], and doctors cannot yet provide sufficient guidance regarding this treatment based on robust evidence of clinical- and cost-effectiveness.

Two studies are frequently cited in reviews of IACIs in JIA and clinical practice guidelines to draw conclusions concerning this intervention [46,47]. These prospective, randomised studies focus on the comparative efficacy of commonly used steroid preparations triamcinolone hexacetonide $(\mathrm{TH})$ and triamcinolone acetonide (TA), concluding that $\mathrm{TH}$ was superior. However, a major limitation of these studies was that a non-treatment, placebo, or sham control arm by which to compare the effects of the IACI interventions was not possible due to ethical constraints. Moreover, participants included were not on stable medication for an appropriate period prior to enrolment, so both studies were vulnerable to confounding bias. New evidence has emerged since previous reviews have been published; however, limitations in these studies are still evident. The well documented restrictions of the commercial availability of $\mathrm{TH}$ resulted in this preparation being replaced by TA in at least one study included in this review [25]. A lack of standardisation of IACI interventions was identified amongst studies retrieved by our search criteria which may be problematic. Frequently studies do not describe their injection technique, which can vary between individual institutes [22]. Moreover, studies included in this review have utilised varying post-injection management strategies including physiotherapy rehabilitation [31], immobilisation [31,34], and a period of non-weight-bearing or avoidance of physical activity $[26,37,40,45]$. Despite this, other studies included did not describe a post-injection strategy $[24,35,38,42,43]$. These approaches appear to lack consensus, and at present there is a lack of evidence available to support a specific post-injection procedure.

A novel finding from this review was that several studies employed image-guidance to aid their IACIs [24-26,38,42]. Ultrasonography-guided injections also have been identified as a useful imaging technique for IACIs as they permit the real-time guidance of the needle and allow confirmation of correct deposition of steroid [24,35]. These studies concluded the use of imaging techniques ensured correct needle placement and guided IACIs were superior to standard 'blinded' injection and aspiration techniques. These studies also raise further questions surrounding imageguidance concerning whether or not image-guidance can reduce the rate of complications associated with IACIs. At present there have been no RCTs comparing guided and blindly administered IACIs in JIA.

The conclusions from this review contribute to the evidence base in support of current JIA management practices through aggressive and early intervention to reduce disease activity [11]. This highlights inconsistencies and evidence gaps concerning the efficacy of IACIs in JIA. It also provides valuable additional and contemporary information through a robust and systematic approach relative to previous reviews. While investigating the efficacy of IACIs, post-injection procedure was included in the study description as a contextual factor. This did not occur in previous reviews. Not all studies included a post-injection technique. Those studies that did include post injection procedure are described in Table 3. However, the lack of consistency among the described injection procedures makes it difficult for comparison to make a consensus. This is an area which requires further research.

There were a number of limitations for this systematic review that should be acknowledged. Language was limited to English which is generally not recommended, but is often difficult to overcome [50]. There were two identified studies which had to be excluded based on language barriers (Spanish and Russian languages) that could have potentially had an impact on the findings of this review. It is acknowledged that a number of studies were identified by hand-searching that were not identified using the detailed search strategy. The original search strategy was reviewed to investigate whether or not it was adequate. The minor problem with the search strategy was subsequently attributed to an indexing issue within the databases that could not be overcome by altering the search strategy. However, in spite of this limitation, the pragmatic nature of the reviewing strategy permitted important and relevant studies which met the inclusion criteria to be identified by hand.

\section{Conclusions}

In conclusion, there is conflicting and inconclusive evidence for the efficacy of lower limb IACIs for JIA. While there is enough quantity among the current body of knowledge to provide weak evidence in some outcome domains for generalised efficacy of IACIs administered to the lower limb, efficacy for duration of relief of synovitis in specific lower limb joints remains inconclusive. It is for this reason we conclude that more high quality studies are needed to definitively determine the efficacy of lower limb IACIs for improving outcomes associated with the JIA disease process. 


\section{Additional file}

\section{Additional file 1: Search strategy.}

\section{Competing interests}

The authors have no competing interests to declare.

\section{Authors' contributions}

$\mathrm{GJH}$ and $\mathrm{HJ}$ were responsible for the conception of the study and all authors contributed to its design. $\mathrm{HJ}$ and $\mathrm{KH}$ were responsible for the acquisition and interpretation of the data with contributions from $\mathrm{GJH}$ where necessary. All authors were involved in drafting the article or revising it critically for important intellectual content and all authors approved the final version to be published.

Received: 20 January 2014 Accepted: 1 June 2014

Published: 11 June 2014

\section{References}

1. Manners PJ, Bower C: Worldwide prevalence of juvenile arthritis- why does it vary so much? Pediatrics 1996, 98(1):84-90.

2. Baszo A, Consolaro A, Ruperto N, Pistorio A, Viola S, Magni-Manzoni S, Malattia C, Buoncompagni A, Loy A, Martini A, Ravelli A: Development and testing of reduced joint counts in juvenile idiopathic arthritis. J Rheumato/ 2009, 36:183-190.

3. Foster HE, Eltringham MS, Kay LJ, Friswell M, Abinun M, Myers A: Delay in access to appropriate care for children presenting with musculoskeletal symptoms and ultimately diagnosed with juvenile idiopathic arthritis. Arthritis Rheum 2007, 57(6):921-927.

4. Jousse-Joulin S, Breton S, Cangemi C, Fenoll B, Bressolette L, de Parsau L, Saraux A, Devauchelle-Pensec V: Ultrasonography for detecting enthesitis in juvenile idiopathic arthritis. Arthritis Care Res 2011, 63(6):849-855.

5. Rooney M, McAllister C, Burns JFT: Ankle disease in juvenile idiopathic arthritis: ultrasound findings in clinically swollen ankles. J Rheumatol 2009, 36:1725-1729.

6. Palmisani E, Solari N, Magni-Manzoni S, Pistorio A, Labo E, Panigada S, Martini A, Ravelli A: Correlation between juvenile idiopathic arthritis activity and damage measures in early, advanced, and longstanding disease. Arthritis Rheum 2006, 55(6):843-849.

7. Ferrari J: A review of foot deformities seen in juvenile chronic arthritis. Foot 1998, 8(4):193-196.

8. Selvaag AM, Flatø B, Dale K, Lein G, Vinje O, Smerdel-Ramoya A, Førre $\varnothing$ : Radiographic and clinical outcome in early juvenile rheumatoid arthritis and juvenile spondyloarthropathy: A 3-year prospective study. J Rheumatol 2006, 33(7):1382-1391.

9. Ramanan AV, Whitworth P, Baildam EM: Use of methotrexate in juvenile idiopathic arthritis. Arch Dis Child 2003, 88:197-200.

10. Hashkes PJ, Laxer RM: Medical treatment of juvenile idiopathic arthritis. JAMA 2005, 294(13):1671-1684.

11. Beresford MW, Baildam EM: New advances in the management of juvenile idiopathic arthritis-2: The era of biologicals. Arch Dis Child Educ Pract Ed 2009, 94(5):151-156.

12. Horneff G, De Bock F, Foeldvari I, Girschick HJ, Michels H, Moebius D, Schmeling $H$, the German and Austrian Paediatric Rheumatology Collaborative Study Group: Safety and efficacy of combination of etanercept and methotrexate compared to treatment with etanercept only in patients with juvenile idiopathic arthritis (JIA): preliminary data from the German JIA Registry. Ann Rheum Dis 2009, 68:519-525.

13. Gartlehner G, Hansen RA, Jonas BL, Thieda P, Lohr KN: Biologics for the treatment of juvenile idiopathic arthritis: a systematic review and critical analysis of the evidence. Clin Rheumatol 2008, 27:67-76.

14. Cleary AG, Murphy HD, Davidson JE: Intra-articular corticosteroid injections in juvenile idiopathic arthritis. Arch Dis Child 2003, 88:192-196.

15. Job-Deslandre C, Menkes CJ: Complications of intra-articular injections of triancinolone hexacetonide in chronic arthritis in children. Clin Exp Rheumatol 1990, 8(4):413-416.

16. Stoustrup P, Kristensen KD, Verna C, Kuseler A, Pedersen TK, Herlin T: Intra-articular steroid injection for temporomandibular joint arthritis in juvenile idiopathic arthritis: a systematic review of efficacy and safety. Semin Arthritis Rheum 2013, 43(1):63-70.
17. Wallen MM, Gillies D: Intra-articular steroids and splints/rest for children with juvenile idiopathic arthritis and adults with rheumatoid arthritis. Cochrane Database Syst Rev 2006, 25(1):CD002824.

18. Higgins JP, Green S: Cochrane for systematic reviews of interventions, version 5.1.0. [www.cochrane-handbook.org]

19. Marti P, Molinari L, Bolt IB, Seger R, Saurenmann RK: Factors influencing the efficacy of intra-articular steroid injections in patients with juvenile idiopathic arthritis. Eur J Pediatr 2008, 167(4):425-430.

20. Dueckers G, Guellac N, Arbogast M, Dannecker G, Foeldvari I, Frosch M, Ganser G, Heiligenhaus A, Horneff G, Illhardt A, Kopp I, Krauspe R, Markus B, Michels H, Schneider M, Singendonk W, Sitter H, Spamer M, Wagner N, Niehues T: Evidence and consensus based GKJR guidelines for the treatment of juvenile idiopathic arthritis. Clin Immunol 2012, 142(2):176-193.

21. Cheng OT, Souzdalnitski D, Vrooman B, Cheng J: Evidence-based knee injections for the management of arthritis. Pain Med 2012, 13(6):740-753.

22. Wallace C: Intra-articular steroid injections in JIA. Reumatologia 2002, 18(3):111-114

23. Garg AX, Hackman D, Tonelli M: Systematic review and meta-analysis: when one study is just not enough. Clin J Am Soc Nephrol 2008, 3(1):253-260.

24. Laurell L, Court-Payen M, Nielsen S, Zak M, Boesen M, Fasth A: Ultrasonography and color Doppler in juvenile idiopathic arthritis: diagnosis and follow-up of ultrasound-guided steroid injection in the ankle region. A descriptive interventional study. Pediatr Rheumatol 2011, 9(4):1-12.

25. Cahill AM, Cho SS, Baskin KM, Beukelman T, Cron RQ, Kaye RD, Towbin RB: Benefit of fluoroscopically guided intraarticular, long-acting corticosteroid injection for subtalar arthritis in juvenile idiopathic arthritis. Pediatr Radiol 2007, 37(6):544-548.

26. Beukelman T, Arabshahi B, Cahill AM, Kaye RD, Cron RQ: Benefit of intraartciular corticosteroid injection under fluoroscopic guidance for subtalar arthritis in juvenile idiopathic arthritis. J Rheumatol 2006 33(11):2330-2336.

27. Hennessy K, Woodburn J, Steultjens MPM: Custom foot orthoses for rheumatoid arthritis. Arthritis Care Res 2012, 64(3):311-320.

28. Ariens GAM, van Mechelen W, Bongers PM, Bouter LM, van der Wal G: Physical risk factors for neck pain. Scand J Work Environ Health 2000, 26(1):7-19.

29. Moher D, Liberati A, Tetzlaff J, Altman DG, PRISMA Group: Preferred reporting items for systematic reviews and meta-analyses: The PRISMA statement. PLos Med 2009, 6(6):e1000097.

30. Balogh Z, Ruzsonyi E: Triamcinolone hexacetonide versus betamethasone: A double-blind comparative study of the long-term effects of intraarticular steroids in patients with juvenile chronic arthritis. Scand J Rheumatol 1988, 1988(67):80-82.

31. Al-wahadneh AM: Intra-articular methylprednisolone acetate in the management of chronic arthritis in children, King Hussein medical centre experience. J Bahrain Med Soc 2005, 17(1):5-9.

32. Allen RC, Gross KR, Laxer RM, Malleson PN, Beauchamp RD, Petty RE: Intraarticular triamcinolone hexacetonide in the management of chronic arthritis in children. Arthritis Rheum 1986, 29(8):997-1001.

33. Earley A, Cuttica RJ, McCullough C, Ansell BM: Triamcinolone into the knee joint in juvenile chronic arthritis. Clin Exp Rheumatol 1986, 64(10):153-155.

34. Fberhard BA, Sison MC, Gottlieb BS, llowite NT: Comparison of the intraarticular effectiveness of triamcinolone hexacetonide and triamcinolone acetonide in treatment of juvenile rheumatoid arthritis. J Rheumatol 2004 2004, 31(12):2507-2512.

35. Eich GF, Halle F, Hodler J, Seger R, Willi UV: Juvenile chronic arthritis: imaging of the knees and hips before and after intraarticular steroid injection. Pediatr Radiol 1994, 24(8):558-563.

36. Hertzberger-ten Cate $R$, de Vries van der Vlugt BCM, van Suijlekom-Smit LWA, Cats A: Intra-articular steroids in pauciarticular juvenile chronic arthritis, type 1. Eur J Pediatr 1991, 150(3):170-172.

37. Honkanen VEA, Rautonen JK, Pelkonen PM: Intra-articular glucocorticoids in early juvenile chronic arthritis. Acta Paediatr 1993, 82(12):1072-1074.

38. Huppertz HI, Tschammier A, Horwitz AE, Schwab KO: Intraarticular corticosteroids for chronic arthritis in children: Efficacy and effects on cartilage and growth. J Pediatr 1995, 127(3):317-321.

39. Lepore L, Del Santo M, Malorgio C, Presani G, Perticarari S, Prodan M Di Leo G, Leone $V$, Tommasini A: Treatment of juvenile idiopathic arthritis with intra-articular triamcinolone hexacetonide: Evaluation of clinical effectiveness correlated with circulating ANA and T $\Upsilon / \delta+$ and B CD5+ lymphocyte populations of synovial fluid. Clin Exp Rheumatol 2002, 20(5):719-722. 
40. Papadopoulou C, Kostik M, Gonzalez-Fernandez MI, Bohm M, NietoGonzalez JC, Pistorio A, Lanni S, Consolaro A, Martini A, Ravelli A: Delineating the role of multiple intraarticular corticosteroid injections in the management of juvenile idiopathic arthritis in the biologic era. Arthritis Care Res 2012, 65(7):1112-1120.

41. Ravelli A, Manzoni SM, Viola S, Pistorio A, Ruperto N, Martini A: Factors affecting the efficacy of intraarticular corticosteroid injection of knees in juvenile idiopathic arthritis. J Rheumato/ 2001, 28(9);2100-2102.

42. Remedios D, Martin K, Kaplan G, Mitchell R, Woo P, Rooney M: Juvenile chronic arthritis: Diagnosis and management of tibio-talar and sub-talar disease. Pediatr Rheumatol 1997, 36(11):1214-1217.

43. Sherry DD, Stein LD, Reed AM, Schanberg LE, Kredich DW: Prevention of leg length discrepancy in young children with pauciarticular juvenile rheumatoid arthritis by treatment with intraarticular steroids. Arthritis Rheum 1999, 42(11):2330-2334.

44. Sornay-Soares C, Job-Deslandre C, Kahan A: Joint lavage for treating recurrent knee involvement in patients with juvenile idiopathic arthritis. Joint Bone Spine 2004 2004, 71(4):296-299.

45. Verma S, Gupta R, Lodha R, Kabra SK: Feasibility and efficacy of intraarticular steroids (IAS) in juvenile idiopathic arthritis (JIA). Indian Pediatrics 2009, 46(3):264-265.

46. Zulian F, Martini G, Gobber D, Agoso C, Gigante C, Zacchello F: Comparison of intra-articular triamcinolone hexacetonide and triamcinolone acetonide in oligoarticular juvenile idiopathic arthritis. Rheumatology (Oxford) 2003, 42 (10):1254-1259.

47. Zulian F, Martini G, Gobber D, Plebani M, Zacchello F, Manners P: Triamcinolone acetonide and hexacetonide intra-articular treatment of symmetrical joints in juvenile idiopathic arthritis: a double-blind trial. Rheumatology (Oxford) 2004, 43(10):1288-1291.

48. Macpherson H: Pragmatic clinical trials. Complement Ther Clin Pract 2004, 12(23):136-140.

49. Australian Government Department of Health and Aging: Medicare Benefits Schedule Book Category 3; [http://www.health.gov.au/internet/mbsonline/ publishing.nsf/Content/5EEB8ED3EDC0FD1ECA257BD50006E5B6/:File/ 201310-Cat3.pdf]

50. Smith V, Devane D, Begley CM, Clarke M: Methodology in conducting a systematic review of systematic review of healthcare interventions. BMC Med Res Methodol 2011, 11(15):1-6.

doi:10.1186/1546-0096-12-23

Cite this article as: Jennings et al:: The clinical effectiveness of intraarticular corticosteroids for arthritis of the lower limb in juvenile idiopathic arthritis: a systematic review. Pediatric Rheumatology 2014 12:23.

\section{Submit your next manuscript to BioMed Central and take full advantage of:}

- Convenient online submission

- Thorough peer review

- No space constraints or color figure charges

- Immediate publication on acceptance

- Inclusion in PubMed, CAS, Scopus and Google Scholar

- Research which is freely available for redistribution 